\title{
2 Collagen VI regulates peripheral nerve regeneration by modulating macrophage recruitment and polarization
}

\author{
4 Peiwen Chen • Matilde Cescon • Gaia Zuccolotto• \\ 5 Lucilla Nobbio - Cristina Colombelli • Monica Filaferro • \\ 6 Giovanni Vitale • M. Laura Feltri • Paolo Bonaldo
}

7 Received: 28 September 2014 / Revised: 15 November 2014 / Accepted: 17 November 2014

8 C) Springer-Verlag Berlin Heidelberg 2014

9 Abstract Macrophages contribute to peripheral nerve 10 regeneration and produce collagen VI, an extracellu11 lar matrix protein involved in nerve function. Here, we 12 show that collagen VI is critical for macrophage migra13 tion and polarization during peripheral nerve regeneration. 14 Nerve injury induces a robust upregulation of collagen $15 \mathrm{VI}$, whereas lack of collagen VI in Col6al ${ }^{-/-}$mice delays 16 peripheral nerve regeneration. In vitro studies demonstrated that collagen VI promotes macrophage migration and polarization via AKT and PKA pathways. Col6al ${ }^{-1-}$ macrophages exhibit impaired migration abilities and reduced antiinflammatory (M2) phenotype polarization, but are prone to skewing toward the proinflammatory (M1) phenotype. In vivo, macrophage recruitment and M2 polarization are impaired in Col6al ${ }^{-/-}$mice after nerve injury. The delayed nerve regeneration of $\mathrm{Colbal}^{-1-}$ mice is induced

Electronic supplementary material The online version of this article (doi:10.1007/s00401-014-1369-9) contains supplementary material, which is available to authorized users.

P. Chen $\cdot$ M. Cescon $\cdot$ P. Bonaldo $(\square)$

Department of Molecular Medicine, University of Padua, Via Ugo Bassi 58/B, 35131 Padua, Italy

e-mail: bonaldo@bio.unipd.it

G. Zuccolotto

Department of Surgery Oncology and Gastroenterology,

University of Padua, 35128 Padua, Italy

\section{Nobbio}

Department of Neurosciences, Rehabilitation, Ophthalmology, Genetics and Mother and Child Sciences, University of Genoa, 16132 Genoa, Italy

C. Colombelli · M. L. Feltri

Department of Genetics and Cell Biology, San Raffaele Scientific Institute, 20132 Milan, Italy by macrophage deficits and rejuvenated by transplantation of wild-type bone marrow cells. These results identify collagen VI as a novel regulator for peripheral nerve regeneration by modulating macrophage function.

Keywords Collagen VI $\cdot$ Nerve regeneration ·

Macrophage $\cdot$ Migration $\cdot$ Polarization $\cdot$ Peripheral nerve

\section{Introduction}

Unlike the central nervous system, axons in the peripheral nervous system (PNS) have the ability to regenerate even after severe injury. Successful peripheral nerve regeneration is a process that requires the concerted interplay of glial cells, growth factors, cell adhesion molecules and extracellular matrix (ECM) proteins, as well as the recruitment of macrophages [21]. Macrophages are critical for the inflammatory response, a process that needs to be tightly controlled

M. Filaferro

Section of Pharmacology, Department of Biomedical, Metabolic

Sciences and Neurosciences, University of Modena and Reggio

Emilia, 41125 Modena, Italy

\section{G. Vitale}

Section of Pharmacology, Department of Life Sciences,

University of Modena and Reggio Emilia, 41125 Modena, Italy

M. L. Feltri

Hunter James Kelly Research Institute, University at Buffalo, State University of New York, New York, NY 14203, USA

\begin{tabular}{|l|lll|}
\hline Journal : Large 401 & Dispatch : 21-11-2014 & Pages : 17 \\
Article No : 1369 & $\square$ LE & $\square$ TYPESET \\
MS Code : ANEU-D-14-00635 & $\square \quad$ CP & $\square \quad$ DISK \\
\hline
\end{tabular}


to avoid excessive tissue damage after injury [31]. Following PNS injury, macrophages not only contribute to debris clearance, growth factor production and ECM remodeling in the distal nerve, but also stimulate regeneration near the axotomized neuronal cell bodies [20, 32, 34]. Leukemia inhibitory factor (LIF), interleukin (IL)- $1 \alpha$, IL- $1 \beta$ and monocyte chemoattractant protein-1 (MCP-1) have been identified as the major regulators for macrophage recruitment after peripheral nerve injury [36, 41, 45]. However, how these factors are modulated during macrophage recruitment remains elusive. Furthermore, additional factors for regulating macrophage migration after peripheral nerve injury need to be identified.

Macrophages exhibit remarkable plasticity and adopt pro- and antiinflammatory phenotypes (M1 and M2, respectively) in response to the stimulation of environmental signals $[4,5,19]$. Indeed, M1 and M2 macrophages exhibit distinct functions, where M1 macrophages stimulate an immune response, and M2 macrophages are immunosuppressive cells promoting tissue repair and remodeling $[4,6,18,29]$. Interestingly, macrophages can undergo dynamic changes between M1 and M2 phenotypes, a process known as polarization skewing [32]. For example, when macrophages are stimulated with lipopolysaccharides (LPS) or interferon (IFN)- $\gamma$, they skew to an M1 phenotype characterized by high expression of inducible nitric oxide synthase (iNOS) and cyclooxygenase (COX)-2. Conversely, macrophages are polarized to an M2 phenotype upon stimulation with IL-4, IL-10 or IL-13, a condition characterized by high expression of mannose receptor $\mathrm{C}$ type 1 (MRC1/CD206), arginase I (Arg-1) and peroxisomal proliferator activated receptor gamma (PPAR $\gamma)$ [4]. Polarization of macrophages toward the M2 phenotype in injury sites by local delivery of IL-4 promotes peripheral nerve regeneration [30]. However, the precise mechanisms governing macrophage polarization, especially in the peripheral nerve injury model, are still incompletely understood.

Collagen VI is a large ECM molecule made of three major genetically distinct chains, $\alpha 1(\mathrm{VI}), \alpha 2(\mathrm{VI})$ and $\alpha 3(\mathrm{VI})$, which are encoded by Col6a1, Col6a2 and Col6a3 genes, respectively [5]. Although our previous studies demonstrated that collagen VI is an essential component of peripheral nerves required for proper nerve myelination and function [7], the role of collagen VI in peripheral nerve regeneration is completely unknown. M2 macrophages produce higher amounts of collagen VI than M1 macrophages [40]. Moreover, collagen VI enhances the adhesion of monocytes [40]. These findings raise the question whether collagen VI is required for macrophage activities, such as migration and polarization. Here, we show that collagen VI is critical for macrophage migration and M2 polarization via AKT and PKA pathways. As a result, peripheral nerve regeneration is strikingly impaired in collagen VI null $\left(\right.$ Col6al $\left.^{-1-}\right)$ mice, where a targeted inactivation of the
Col6al gene blocks the assembly and secretion of collagen VI $[2,23]$. These findings provide novel mechanistic data for macrophage activity and plasticity and demonstrate that collagen VI is a key regulator of PNS regeneration through modulation of macrophage function.

\section{Materials and methods}

Animals

Col6al $^{+/+}$(wild-type) and Col6a1 ${ }^{-/-}$mice in the C57BL/6 background were used in this study [2, 23]. All in vivo experiments were performed in 6-7-month-old mice. Native collagen VI protein was purified from newborn mice as previously described [23]. Animal procedures were approved by the Ethics Committee of the University of Padua and authorized by the Italian Ministry of Health.

\section{Surgical procedures}

Mice were anesthetized with ketamine $(100 \mathrm{mg} / \mathrm{kg}$ body weight) and xylazine ( $8 \mathrm{mg} / \mathrm{kg}$ body weight), and the right sciatic nerve was exposed and crushed with a liquid nitrogen-cooled Dumont forceps for $20 \mathrm{~s}$, stopped for a $10 \mathrm{~s}$ interval and then subjected to a second crush at the same site. The crush site was about $45 \mathrm{~mm}$ from the tip of the third digit, which was labeled with India ink.

Macrophage depletion

Macrophages were depleted by i.p. injection of clodronate liposome (ClodronateLiposomes.com) in mice as reported previously [16]. Briefly, clodronate liposome (200 $\mu \mathrm{l} /$ mouse) was injected at $1,3,5$ and 8 days post-crush to obtain macrophage-depleted mice. Control mice received an equal volume of PBS liposome at the same time points.

Bone marrow transplantation

The bone marrow transplantation was performed as described previously [12]. Briefly, bone marrow was harvested from 6- to 8-week-old wild-type mice by flushing the femurs and tibias with $2 \%$ fetal bovine serum in phosphate-buffered saline. Cells $\left(2 \times 10^{6}\right)$ were intravenously injected through the tail vein into lethally irradiated (10 Gy) 4-month-old wild-type and Col6a1 ${ }^{-1-}$ mice. Sciatic nerve injury was performed 6 weeks after the transplantation.

Functional tests

Prior to and after crush, nontoxic paint was applied to the hindpaws, and the mice were allowed to walk on a white

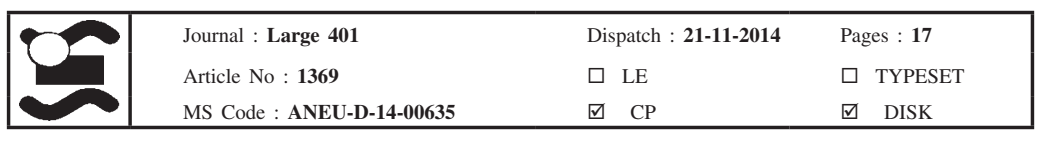


paper. From the footprint gait, the parameters of print length (the distance between the heel and the third toe, abbreviated as PL) and toe spread (the distance from the first toe to the fifth toe, abbreviated as TS) from both the normal side $(\mathrm{N})$ and experimental side $(\mathrm{E})$ were recorded. The sciatic functional index (SFI) was calculated with the following formula: SFI $=118.9$ [(ETS-NTS $) / \mathrm{NTS}]-51.2$ [(EPL-NPL)/NPL] -7.5 as previously reported [22, 52]. Analysis of the toe spread reflex and toe pinch was carried out as previously described [43].

\section{Histology}

Prior to injury and at 7,21 and 29 days following crush injury, 3 mice of each group were perfused with $4 \%$ paraformaldehyde, the 3-mm distal portion of sciatic nerves was dissected into 1-mm segments and postfixed in $2 \%$ glutaraldehyde for $24 \mathrm{~h}$ at $4{ }^{\circ} \mathrm{C}$. Samples (3 mm distal to the site of injury) were osmicated in $2 \%$ osmium tetroxide for $2 \mathrm{~h}$ at room temperature, dehydrated in ascending acetone and embedded in Epon E812 resin (Sigma). Semithin sections $(0.5 \mu \mathrm{m})$ were cut using an Ultracut 200 microtome (Leica) and stained with alkaline toluidine blue. Myelinated axon numbers were analyzed on eight sections per sciatic nerve. For electron microscopy, ultrathin sections $(80 \mathrm{~nm})$ were cut, mounted on copper grids, and stained with uranyl citrate and lead citrate. Grids were observed and photographed on an FEI Tecnai 12 transmission electron microscope. Phagocytic macrophages were identified by the "foamy" morphology, which is induced by the presence of end products of myelin/lipid degradation, as described in previous studies [26].

\section{Matrigel plug assay}

A total of $500 \mu 1$ growth factor-reduced Matrigel (Gibco) supplemented with PBS, 2 \% FBS, purified collagen VI $(500 \mathrm{ng} / \mathrm{ml})$, purified collagen I (Sigma, $500 \mathrm{ng} / \mathrm{ml}$ ) or MCP-1 (ImmunoTools, $10 \mathrm{ng} / \mathrm{ml}$ ) was injected subcutaneously into wild-type and Col6al ${ }^{-/}$mice. After 7 days, the Matrigel plug was harvested and processed for immunofluorescence.

\section{Cell cultures}

The J774 macrophage cell line was purchased from American Type Culture Collection and cultured in Dulbecco's modified Eagle's medium (DMEM; Gibco) containing $10 \%$ fetal bovine serum (FBS, Gibco), $0.2 \mathrm{M}$ L-glutamine (Invitrogen) and 1:100 penicillin-streptomycin (Invitrogen). J774 cells were treated with purified native collagen VI for further studies. Primary bone marrow-derived macrophages (BMDMs) and peritoneal macrophage (PMs) were isolated and cultured as previously described with minor modifications [8, 25, 52]. Briefly, for BMDM isolation and culture, 2-3-month-old mice were killed, and bone marrow cells collected by flushing both femurs and tibias with culture medium. Red blood cells were removed using a lysis buffer $\left(150 \mathrm{mM} \mathrm{NH}_{4} \mathrm{Cl}, 0.1 \mathrm{mM}\right.$ $\mathrm{Na}_{2}$ EDTA and $1 \mathrm{mM} \mathrm{KHCO}$, pH 7.2) for $10 \mathrm{~min}$ at room temperature. The remaining cells were then differentiated with $30 \mathrm{ng} / \mathrm{ml}$ M-CSF (ImmunoTools) in DMEM containing $20 \%$ FBS, $0.2 \mathrm{M}$ L-glutamine and 1:100 penicillin-streptomycin for 1 week until the cells reached confluence. For PM isolation and culture, $3 \%$ thioglycolate broth (Sigma) was injected intraperitoneally to induce peritonitis in 2-3-monthold mice. Three days later, peritoneal cells were collected and cultured in DMEM containing $10 \%$ FBS, $0.2 \mathrm{M} \mathrm{L-glu-}$ tamine and 1:100 penicillin-streptomycin. Two hours later, nonadherent cells were removed by washing with PBS, and adherent macrophages were used for further studies. BMDMs and PMs were differentiated into the M2 or M1 phenotype with $20 \mathrm{ng} / \mathrm{ml} \mathrm{IL-4} \mathrm{(ImmunoTools)} \mathrm{or} 5 \mathrm{ng} / \mathrm{ml}$ LPS (Sigma), respectively, for $24 \mathrm{~h}$.

Migration assay

Macrophage migration was assessed using transwell inserts with $5-\mu \mathrm{m}$ pores (Millipore). Briefly, J774 macrophages $\left(2 \times 10^{4}\right.$ cells per well) were seeded into the upper chamber of a transwell filter with DMEM. The same culture medium and purified collagen VI $(0.5$ or $1 \mu \mathrm{g} / \mathrm{ml})$, purified collagen I $(1 \mu \mathrm{g} / \mathrm{ml})$ or MCP-1 $(10 \mathrm{ng} / \mathrm{ml})$ were added to the lower chamber. When indicated, cells were treated with AKTi (Sigma, $10 \mu \mathrm{M}$ ) or H89 (Sigma, $30 \mu \mathrm{M}$ ). Cells were allowed to migrate for $8 \mathrm{~h}$ at $37{ }^{\circ} \mathrm{C}$ and $5 \% \mathrm{CO}_{2}$. After being fixed and stained with ethanol and $0.05 \%$ crystal violet (Sigma), the migrated cells were counted in eight different areas under a light microscope.

Scratch assay

A wound was made in confluent monolayers of J774 cells grown on six-well cell culture plates by scraping with a sterile $200-\mu 1$ pipette tip. The cells were gently rinsed with PBS and further cultured in the presence or absence of purified collagen VI $(1 \mu \mathrm{g} / \mathrm{ml})$, purified collagen I $(1 \mu \mathrm{g} /$ $\mathrm{ml})$, MCP-1 $(10 \mathrm{ng} / \mathrm{ml})$, AKTi $(10 \mu \mathrm{M})$ or H89 $(30 \mu \mathrm{M})$. Images of the cultures were taken immediately after scratching and after $8 \mathrm{~h}$. The migration distances of the macrophages were measured and analyzed using ImagePro Plus 6.0 software (Media Cybernetics).

RNA isolation and real-time RT-PCR

Total RNA from mouse sciatic nerves ( $3 \mathrm{~mm}$ distal to injury site) was isolated using Trizol reagent (Life Technologies)
201

\begin{tabular}{|c|c|c|}
\hline Journal : Large 401 & Dispatch : 21-11-2014 & Pages : 17 \\
\hline Article No : $\mathbf{1 3 6 9}$ & $\square$ LE & $\square$ TYPESET \\
\hline MS Code : ANEU-D-14-00635 & $\nabla \quad \mathrm{CP}$ & DISK \\
\hline
\end{tabular}


following the manufacturer's instructions; $200 \mathrm{ng}$ of total RNA was used to make cDNA using the Superscript II kit (Invitrogen). Quantitative PCR was carried out using the LightCycler 480 system (Roche). The expression level of each gene was calculated by comparing with the Gapdh housekeeping gene. Primers used in this study are shown in Supplementary Table 1.

\section{Immunofluorescence}

After mice had been perfused with $4 \%$ paraformaldehyde, a 3-mm length of sciatic nerve distal to the crush site was removed and postfixed for $4 \mathrm{~h}$ at $4{ }^{\circ} \mathrm{C}$. Tissues or Matrigel plugs were then transferred into $30 \%$ sucrose overnight for cryoprotection. Samples of $10 \mu \mathrm{m}$ were cut in a cryostat (Leica). After blocking with $10 \%$ goat serum for $1 \mathrm{~h}$, sections were incubated with primary antibodies (1:200 dilution) for $2 \mathrm{~h}$ at room temperature or overnight at $4{ }^{\circ} \mathrm{C}$. Primary antibodies against the following proteins were used: $\alpha 3(\mathrm{VI})$ collagen (guinea pig polyclonal, a gift of Raimund Wagener, Cologne, Germany) [15]; $\beta$-III tubulin (mouse monoclonal, Sigma); CD68, F4/80 (rat monoclonal, AbD Serotec); CD206 (rabbit polyclonal, Abcam); MAG (rabbit monoclonal, Cell Signaling). The samples were then transferred to secondary antibodies (1:200 dilution) and Hoechst 33258 (Sigma) for $1 \mathrm{~h}$ at room temperature. The following secondary antibodies were used: anti-rat CY3, anti-rabbit CY3 and anti-guinea-pig CY2 (Jackson Immunoresearch). After washing three times in PBS, slides were mounted using $80 \%$ glycerol.

\section{Western blotting}

Mice were killed by cervical dislocation, and sciatic nerves ( $3 \mathrm{~mm}$ distal to the crush site) were removed and frozen in nitrogen immediately. The tissues or cells were homogenized in lysis buffer (Millipore) with phosphatase inhibitors (Sigma) and protease inhibitors (Roche, Basel, Switzerland). The protein concentration was determined by BCA assay (Thermo Sciemtific). Samples of $20 \mu \mathrm{g}$ protein were applied to SDS-PAGE gels (Invitrogen) and blotted onto a PDVF membrane (Millipore). Membranes were incubated with primary antibodies (1:1,000 dilution) overnight at $4{ }^{\circ} \mathrm{C}$. Primary antibodies against the following proteins were used for Western blot analysis: $\alpha 1(\mathrm{VI})$ collagen, Arg-1, CD206, iNOS (rabbit polyclonal, Santa Cruz Biotechnology); $\beta$-actin (mouse monoclonal, Sigma); AKT, phospho-PKA, PKA (rabbit polyclonal, Cell Signaling); phospho-AKT, COX-2, MAG, PPAR $\gamma$ (rabbit monoclonal, Cell Signaling); CD16 (rabbit monoclonal, Abcam); CD206 (rabbit polyclonal, Abcam); CD68 (rat monoclonal, AbD Serotec). After washing three times with TBST, membranes were incubated with HRP-conjugated secondary antibodies (1:1,000 dilution; Amersham Bioscience) for $1 \mathrm{~h}$ at room temperature. Detection was by chemiluminescence (Pierce). The panels show representative images of two separate protein extracts derived from two different mice. Densitometric quantification was performed by Image-Pro Plus 6.0 software (Media Cybernetics).

Statistical analysis

Data are represented as mean \pm SEM. Statistical analysis of data was carried out using Student's $t$ test, except for the analysis of the toe spread reflex in PBS- and clodronate-liposome-treated mice, where the chi-square test was used, and the analysis of collagen VI mRNA expression after injury, where one-way ANOVA followed by post hoc tests was used. $P<0.05$ was considered as a significant difference.

\section{Results}

Expression of collagen VI is increased after sciatic nerve crush injury

To explore the role of collagen VI in PNS regeneration, we first examined whether collagen VI expression is upregulated upon sciatic nerve crush injury in adult mice. Realtime RT-PCR showed that the levels of Col6al and Col6a3 transcripts were increased at 7 and 14 days post-injury, whereas the levels of Col6a2 transcripts started to increase within 3 days after sciatic nerve crush, and the expression of all three mRNAs reached a peak between 3 and 7 days post-injury (Fig. 1a-c). Western blot analysis for $\alpha 1$ (VI) and $\alpha 2$ (VI) chains (Fig. 1d) and immunofluorescence for $\alpha 3$ (VI) chain (Fig. 1e, f) showed that the protein levels for collagen VI were also increased between 3 and 7 days post-injury. Taken together, these data indicate that crush injury of the sciatic nerve induces a robust upregulation of both mRNA and protein levels of collagen VI, pointing to a potential role for this molecule during PNS regeneration.

\section{Collagen VI is required for peripheral nerve regeneration}

Wallerian degeneration is a process that includes the breakdown of axons and phagocytosis of damaged axons and myelin debris after injury, which is strictly required for axon regeneration [36]. We therefore first examined Wallerian degeneration in collagen VI-deficient mice. Toluidine blue staining and electron microscopy showed that at 7 days post-injury sciatic nerves from wild-type mice had advanced signs of myelin breakdown and a high incidence of phagocytic macrophages. However, both of these features were noticeably lower in $\mathrm{Colba1}^{-/-}$mice

\begin{tabular}{|llll|} 
Journal : Large 401 & Dispatch : 21-11-2014 & Pages : 17 \\
Article No : $\mathbf{1 3 6 9}$ & $\square$ LE & $\square$ TYPESET \\
MS Code : ANEU-D-14-00635 & $\square \quad$ CP & $\square$ DISK \\
\hline
\end{tabular}



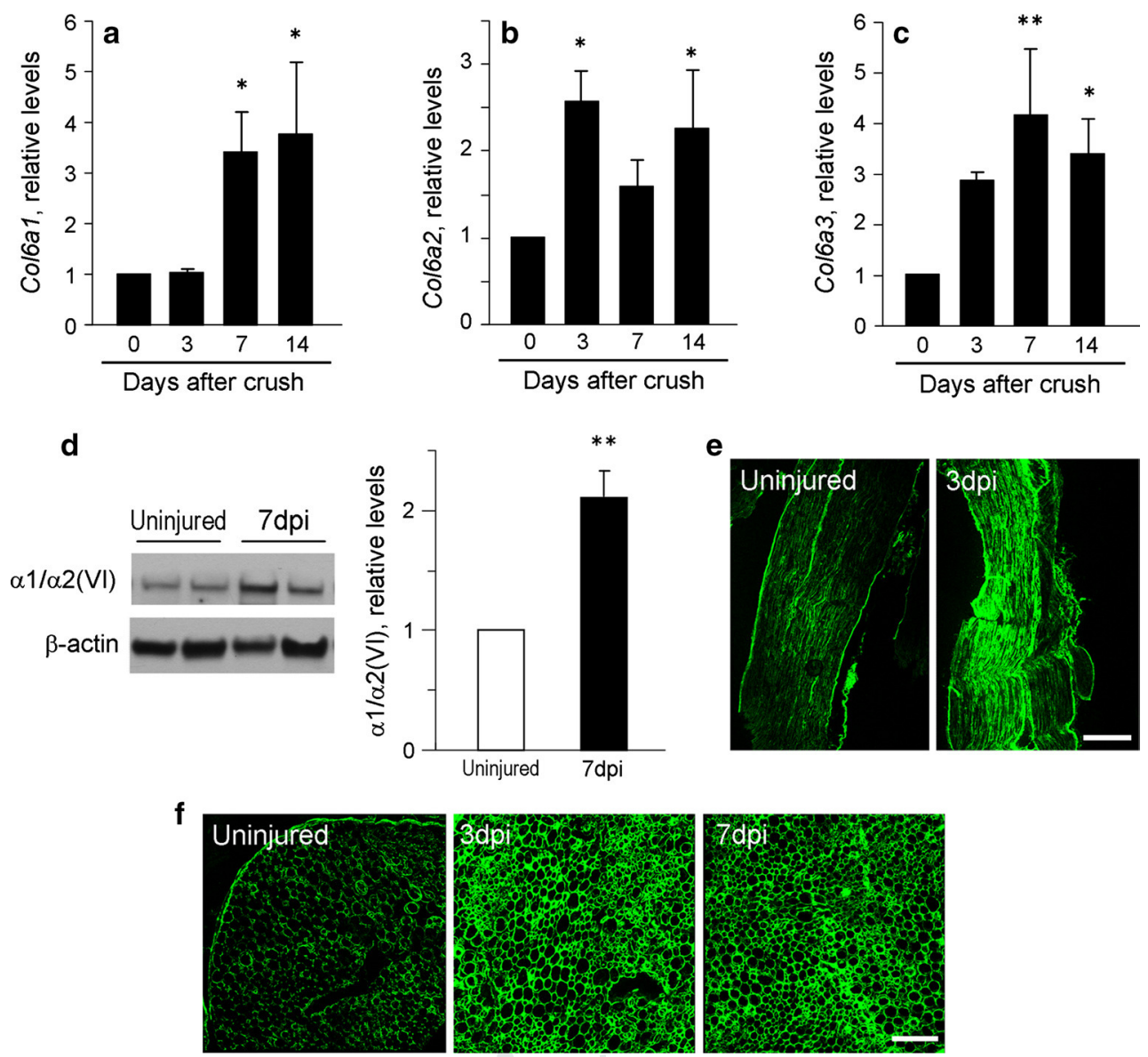

Fig. 1 Collagen VI expression is enhanced upon peripheral nerve crush. Real-time RT-PCR for Col6a1 (a), Col6a2 (b) and Col6a3 (c) in uninjured (0) or injured sciatic nerves at 3, 7 and 14 days postcrush $(n=3-5)$. There was a statistically significant difference among groups as determined by one-way ANOVA analysis of Col6al $[F(3,13)=3.739, P=0.039]$, Col6a2 $[F(3,13)=3.506, P=0.046]$ and Col6a3 $[F(3,13)=4.600, P=0.021]$. A post hoc test revealed that the relative levels of Col6a1 or Col6a3 were statistically significantly increased in injured nerves at $7 \mathrm{dpi}(3.46 \pm 0.77, P=0.035$, or $4.15 \pm 1.29, P=0.004)$ and $14 \mathrm{dpi}(3.76 \pm 1.44, P=0.018$, or $3.38 \pm 0.68, P=0.033)$, but not at 3 dpi $(1.04 \pm 0.05, P=0.973$, or $2.85 \pm 0.17, P=0.087$ ), when compared to uninjured nerves, and the relative levels of Col6a2 were statistically significantly increased in injured nerves at $3 \mathrm{dpi}(2.55 \pm 0.35, P=0.014)$ and $14 \mathrm{dpi}$ $(2.24 \pm 0.68, P=0.028)$, but not at $7 \mathrm{dpi}(1.59 \pm 029, P=0.271)$,
(Supplementary Fig. S1a, d). Quantitative analysis confirmed that $\mathrm{Col6al}^{-/-}$nerves had more myelinated axons and fewer phagocytic macrophages than wild-type nerves at 7 days post-injury (Supplementary Fig. S1b, c). In keeping with the concept that clearance of myelin debris from injured nerves is necessary for PNS regeneration [14, 47], more myelin was present in Col6al $^{-/-}$nerves than in wildtype nerves at 7 days post-injury (Supplementary Fig. S1a, d). Axonal growth inhibitors, such as myelin-associated glycoprotein (MAG), are usually present in myelin debris after nerve injury [39, 51]. Immunofluorescence and when compared to uninjured nerves. There was no statistically significant difference among the 3-, 7- and 14-dpi groups for Col6a2 $(P=0.415)$ and Col6a3 $(P=0.639)$. Col6al was significantly increased in injured nerves at $14 \mathrm{dpi}$ compared to $3 \mathrm{dpi}(P=0.043)$, but no significant differences were seen between the 7- and 14-dpi $(P=0.758)$ and the 3 - and 7-dpi $(P=0.072)$ groups. d Left panel Western blot analysis for $\alpha 1 / \alpha 2$ (VI) in uninjured sciatic nerves or injured nerves at 7 days post-crush. Right panel Densitometric quantification of $\alpha 1 / \alpha 2$ (VI) vs. actin as determined by three independent Western blot experiments $(n=4 ; * * P<0.01)$. e Immunofluorescence for $\alpha 3(\mathrm{VI})$ in longitudinal sections of uninjured sciatic nerves and injured nerves at 3 days post-crush. Scale bar $250 \mu \mathrm{m}$. f Immunofluorescence for $\alpha 3$ (VI) in cross sections of uninjured sciatic nerves and injured nerves at 3 and 7 days post-crush. Scale bar $50 \mu \mathrm{m} . d p i$ Days post-injury

Western blot analysis revealed that MAG reactivity was higher in $\mathrm{Col6al}^{-/-}$nerves than wild-type nerves at 7 days post-injury (Supplementary Fig. S1e, f). These data support the potential role of collagen VI in Wallerian degeneration following injury.

We then performed experiments to assess whether the inhibited Wallerian degeneration of $\mathrm{Col6al}^{-/-}$mice influences PNS regeneration. First, we measured the sciatic functional index to evaluate the recovery of sensory motorAQ1 1 coordination [24] in mice of both genotypes. As shown in 342 Fig. 2a, the sciatic functional index score was not different 343

\begin{tabular}{|l|lll|}
\hline Journal : Large 401 & Dispatch : 21-11-2014 & Pages : 17 \\
Article No : $\mathbf{1 3 6 9}$ & $\square$ LE & $\square$ TYPESET \\
MS Code : ANEU-D-14-00635 & $\square \quad C P$ & $\square$ DISK \\
\hline
\end{tabular}


Fig. 2 Lack of collagen

VI impairs peripheral nerve regeneration. a Quantification of sensory-motor function of wild-type and Col6a1 ${ }^{-1-}$ mice by analyzing the sciatic functional index from the footprint track before crush and at 7 , $11,14,17,21$ and 29 days post-crush $(n=7 ; * P<0.05$; $* * P<0.01)$. b Quantification of sensory function of wild-type and Col6a1 ${ }^{-1-}$ mice after sciatic nerve crush by recording the initial response time (day post-injury) to the pinch using forceps in the digits 3,4 and $5(n=7 ; * P<0.05$; $* * P<0.01)$. c Quantification of motor function of wild-type and Col6a1 ${ }^{-1-}$ mice after sciatic nerve crush by recording the initial extension time (day postinjury) to toe spreading reflex $(n=7$; $* P<0.05)$. d Representative images of toluidine blue staining and morphometric analysis of the myelinated axon number in cross sections of sciatic nerves from wild-type and Col6a1 ${ }^{-1-}$ mice under uninjured conditions and at 21 and 29 days post-crush $(n=3$; $* * P<0.01)$. Scale bar $40 \mu \mathrm{m}$. dpi days post-injury, WT wild type a

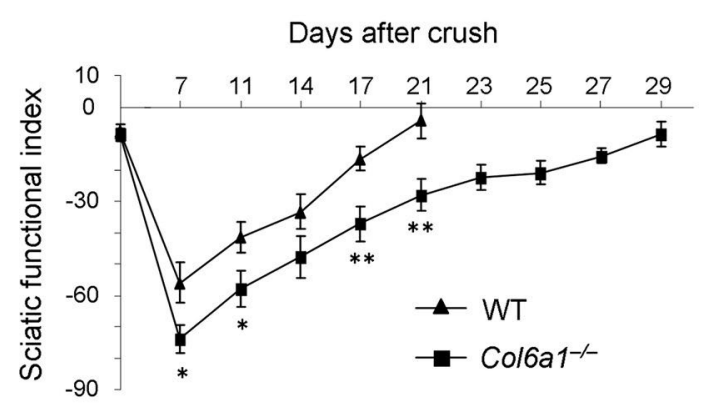

Toe pinch

b

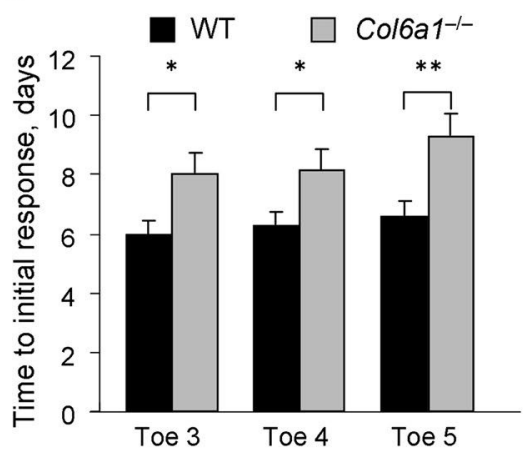

c Toe spread reflex

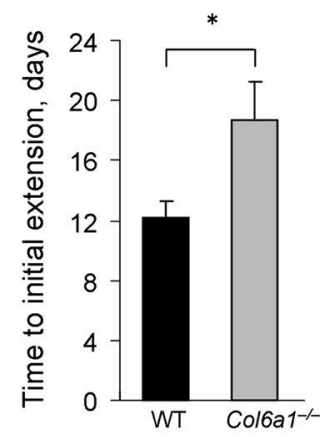

d

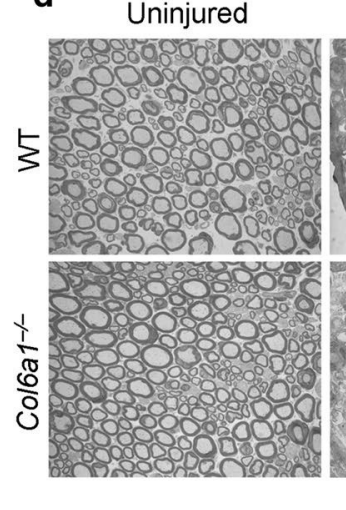

$21 \mathrm{dpi}$

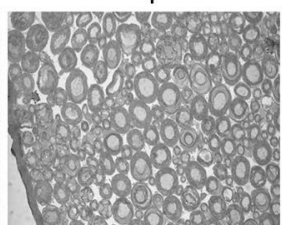

29dpi

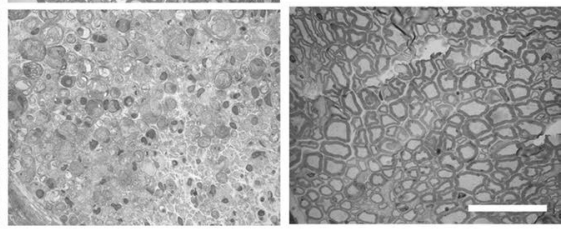

WT $\square$ Col6a1-/-

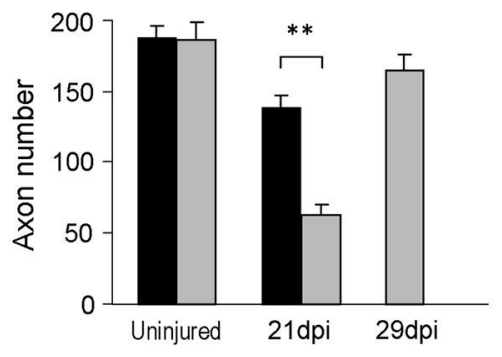

between wild-type and Col6al ${ }^{-/-}$mice before nerve crush. In contrast, the sciatic functional index of $\mathrm{Colbal}^{-/-}$mice was significantly lower than that of wild-type mice at 7 days after injury. This parameter remained significantly lower in collagen VI-deficient mice during the following time points, and a complete functional recovery was observed at 21 and 29 days post-injury in wild-type and Col6al ${ }^{-/-}$ mice, respectively (Fig. 2a). To measure sensory functions, we recorded the response to toe pinch in digits 3,4 and 5 of the crushed hindlimb because they are the main digits innervated by nerves for sensory functions [26]. The time to initial response to the stimuli after sciatic nerve crush was significantly longer in Col6al ${ }^{-1-}$ mice than wild-type mice (Fig. 2b). Next, we utilized the toe spread reflex to evaluate motor function and found that the time to initial toe extension after nerve injury was significantly increased in Col6al $^{-1-}$ mice when compared to wild-type mice (Fig. 2c). Toluidine blue staining showed that the number of myelinated axons was significantly lower in Col6a1 ${ }^{-/-}$ nerves than in wild-type nerves at 21 days post-injury, whereas there was no difference between the two genotypes in uninjured conditions (Fig. 2d). In agreement with the sciatic functional index, the number of myelinated axons was almost completely restored at 21 days post-injury in wildtype mice, while this required 29 days in $\mathrm{Colbal}^{-/-}$mice (Fig. 2d). Altogether, these findings indicate that lack of collagen VI delays peripheral nerve regeneration after injury.

Collagen VI stimulates macrophage migration in vitro and in vivo

In the PNS, macrophages are critical for the removal of debris and contribute to nerve regeneration [13, 51]. To determine whether collagen VI is critical for macrophage activities, we performed in vitro and in vivo experiments to analyze macrophage migration. Transwell assay showed that

\begin{tabular}{|l|ll|} 
Journal : Large 401 & Dispatch : 21-11-2014 & Pages : 17 \\
Article No : $\mathbf{1 3 6 9}$ & $\square$ LE & $\square$ TYPESET \\
MS Code : ANEU-D-14-00635 & $\square \quad$ CP & $\square \quad$ DISK \\
\hline
\end{tabular}


addition of purified collagen VI to the culture medium in the lower chambers at the concentration of $1 \mu \mathrm{g} / \mathrm{ml}$ significantly increased the number of macrophages that had migrated (Fig. 3a and Supplementary Fig. S2a). Scratch assay revealed that collagen VI promotes macrophage motility after scratching, as demonstrated by the markedly enhanced migration distance when macrophages were treated with collagen VI (Fig. 3b and Supplementary Fig. S2b). To investigate the in vivo chemoattractant ability of collagen VI, we used a Matrigel plug assay to examine macrophage density in the Matrigel implanted subcutaneously into wild-type mice. Immunofluorescence for CD68 and F4/80 showed that both these macrophage markers were markedly increased in wild-type mice treated with Matrigel plugs supplemented with purified collagen VI compared to mice treated with PBS-supplemented Matrigel plugs (Fig. 3c and Supplementary Fig. S2c). In addition, we utilized collagen I and MCP-1 as negative and positive controls, respectively, and found that MCP-1, but not collagen I, significantly enhanced macrophage migration in transwell, scratch and Matrigel plug assays (Fig. 3a-c and Supplementary Fig. S2a, b). Next, we used a different experimental setting, where Matrigel plugs supplemented with $2 \%$ FBS were subcutaneously injected in wild-type and Col6a1 ${ }^{-1-}$ mice. Immunofluorescence for the F4/80 marker showed that the macrophage migration capability was dramatically impaired in Col6al ${ }^{-/-}$mice compared to wild-type animals (Fig. 3d), suggesting that in addition to as a chemokine itself, collagen VI is required for other factors inducing macrophage migration.

It has been demonstrated that the AKT and PKA pathways are necessary for macrophage migration $[10,11]$. We thus investigated whether collagen VI-induced macrophage migration is regulated by these signals. Western blot analysis showed that the addition of collagen VI to in vitro macrophages promoted AKT and PKA phosphorylation (Supplementary Fig. S3a, b). Transwell assay demonstrated that the collagen VI-induced increase in the number of migrated macrophages was inhibited by pretreatment with AKT inhibitor (AKTi) or H89, a PKA inhibitor (Fig. 3a and Supplementary Fig. S2a). Moreover, the scratch assay revealed that pretreatment with AKTi or H89 decreased the collagen VI-induced macrophage migration distance (Fig. 3b and Supplementary Fig. S2b). Taken together, these data indicate that collagen VI promotes macrophage migration by regulating the AKT and PKA pathways.

\section{Ablation of collagen VI leads to impaired macrophage} recruitment to the injured nerve

Given the robust chemoattractant activity of collagen VI for macrophages, we further investigated whether the delayed myelin clearance and PNS regeneration are the result of impaired macrophage recruitment in Col6al ${ }^{-/-}$injured nerves.
Immunofluorescence showed that more CD68- and F4/80positive macrophages were present in the injured nerves of wild-type mice than Col6a1 ${ }^{-/-}$mice (Fig. 4a-c). Western blot analysis confirmed that although CD68 was enhanced in both genotypes at 7 days post-injury, the CD68 levels of injured $\mathrm{Col}_{\mathrm{Cal}}{ }^{-/-}$nerves were significantly lower than those of injured wild-type nerves (Fig. 4d). These data indicate that lack of collagen VI impairs macrophage accumulation in injured nerves.

Chemokines and cytokines are important mediators of the immune response. Among them, IL-1 $\beta$ and MCP-1 are two prominent regulators of macrophage recruitment in injured peripheral nerves [36, 41, 45]. We therefore examined whether the impaired macrophage recruitment in Col6a1 ${ }^{-/-}$mice after injury was paralleled by a lower abundance of these two inflammatory regulators. Real-time RT-PCR revealed that although the expression of IL-1 $\beta$ and MCP-1 mRNA was upregulated in both wild-type and Col6al ${ }^{-/-}$nerves at 1 day after crush, the levels of the two transcripts were significantly lower in injured Col6al ${ }^{-1-}$ nerves compared to injured wild-type nerves (Supplementary Fig. S4a, b). These results suggest that in addition to its chemoattractant activity for macrophage migration, collagen VI also affects other inflammatory mediators in injured peripheral nerves.

Collagen VI is critical for macrophage polarization

Peripheral nerve regeneration not only depends on macrophage density, but also requires macrophage polarization toward the M2 phenotype [30]. To investigate the potential role of collagen VI in macrophage polarization, we isolated primary bone marrow-derived macrophages (BMDMs) and peritoneal macrophages (PMs) from wild-type and $\mathrm{Col}_{6} \mathrm{I}^{-/-}$mice and differentiated them toward the M2 and M1 phenotypes with IL-4 and LPS, respectively. Western blot analysis for M2 markers showed that the protein levels of Arg-1, CD206 and PPAR $\gamma$ were increased in wild-type, but not in $\mathrm{Col} \mathrm{al}^{-/-}$BMDMs upon stimulation with IL-4 (Fig. 5a). Similar results were found in PMs, where Arg-1 and PPAR $\gamma$ levels were upregulated in wild-type cells upon stimulation of IL-4, whereas this enhancement was prevented in Col6al $^{-1-}$ cells (Fig. 5b). Furthermore, the defective response of $\mathrm{Collal}^{-/-} \mathrm{PMs}$ was reversed when cells were cultured in the presence of purified collagen VI (Fig. 5b). These results indicate that collagen VI is required for macrophage M2 polarization.

Since collagen VI expression is reduced when macrophages are subjected to M1 stimuli [40], we hypothesized that this reduction may be essential for M1 polarization. Upon LPS stimulation, Col6a1 ${ }^{-/}$PMs displayed a marked enhancement of COX-2, a M1 marker, when compared to wild-type PMs (Fig. 5c). A similar response was found in BMDMs, since upon LPS stimulation Col6al ${ }^{-/-}$cells exhibited higher

\begin{tabular}{|c|c|c|c|}
\hline & Journal : Large 401 & Dispatch : 21-11-2014 & Pages : 17 \\
\hline & Article No : 1369 & $\square$ LE & $\square$ TYPESET \\
\hline & MS Code : ANEU-D-14-00635 & $\nabla \quad C P$ & DISK \\
\hline
\end{tabular}



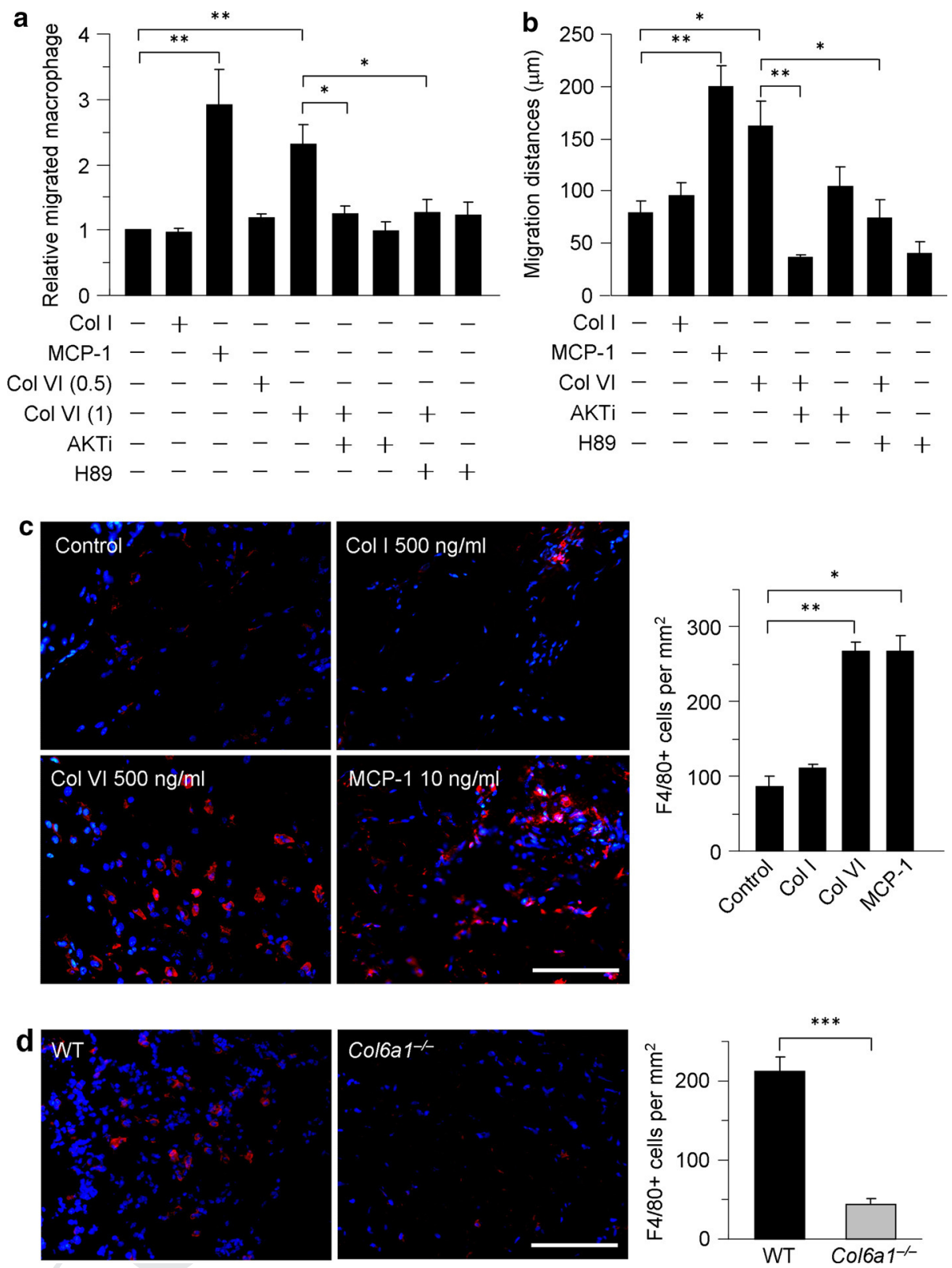

Fig. 3 Collagen VI promotes in vitro and in vivo macrophage migration. a Quantification of migrated J774 macrophages in transwell migration assays upon treatment with PBS (control), collagen VI ( 0.5 or $1 \mu \mathrm{g} / \mathrm{ml}$ ), collagen I or MCP-1. AKTi or H89 was added where indicated $(n=3-4 ; * P<0.05 ; * * P<0.01)$. b Quantification of mean migration distances of J774 macrophages upon treatment with PBS (control), collagen VI $(1 \mu \mathrm{g} / \mathrm{ml})$, collagen I or MCP-1 and subjected to a scratch assay. AKTi or H89 was added where indicated. Cells were analyzed $8 \mathrm{~h}$ after the scratch $(n=3-4$; $* P<0.05$; ** $P<0.01)$. c Left and middle panels Representative images of immunofluorescence for F4/80 in growth factor-reduced Matrigel plugs supple-

enhancement of several M1 markers, such as iNOS, CD16 and COX-2, when compared to wild-type cells (Fig. 5d, e). Of note, these enhancements in Col6al ${ }^{-/-}$cells were partially rescued when cells were cultured in the presence of purified mented with PBS, collagen VI, collagen I or MCP-1 subcutaneously injected into wild-type mice. Scale bar $100 \mu \mathrm{m}$. Right panel Quantitative analysis of migrated F4/80-positive macrophages in Matrigel plugs $(n=3 ; * P<0.05 ; * * P<0.01)$. d Left and middle panels Representative images of immunofluorescence for F4/80 in growth factorreduced Matrigel plugs supplemented with $2 \%$ FBS subcutaneously injected into wild-type (left) and Col6a1 ${ }^{-1-}$ (middle) mice. Scale bar $100 \mu \mathrm{m}$. Right panel Quantitative analysis of migrated F4/80-positive macrophages in Matrigel plugs $(n=3$; $* * * P<0.001)$. AKTi AKT inhibitor, Col I Collagen I, Col VI collagen VI, WT wild type

collagen VI (Fig. 5d, e). These results indicate that collagen VI inhibits macrophage M1 polarization.

To further confirm the effect of collagen VI in macrophage polarization, we cultured $\mathbf{J} 774$ macrophages and

\begin{tabular}{|c|c|c|c|}
\hline & Journal : Large 401 & Dispatch : 21-11-2014 & Pages : 17 \\
\hline & Article No : $\mathbf{1 3 6 9}$ & $\square$ LE & $\square$ TYPESET \\
\hline & MS Code : ANEU-D-14-00635 & $\nabla \quad \mathrm{CP}$ & DISK \\
\hline
\end{tabular}


Fig. 4 Lack of collagen VI leads to impaired of macrophage recruitment into injured nerves. a Immunofluorescence for CD68 in longitudinal sections of sciatic nerves from wild-type and Col6a1 ${ }^{-1-}$ mice under uninjured conditions and at 3 days post-crush. Scale bar $250 \mu \mathrm{m}$. b Immunofluorescence for CD68 in cross sections of sciatic nerves from wild-type and $\mathrm{Col}_{6 a 1^{-1-}}$ mice under uninjured conditions and at 7 days post-crush. Scale bar $50 \mu \mathrm{m}$. c Immunofluorescence for F4/80 in cross sections of sciatic nerves from wild-type and Col6a1 ${ }^{-1-}$ mice under uninjured conditions and at 7 days post-crush. Scale bar $50 \mu \mathrm{m}$. d Left panel Western blot analysis for CD68 in sciatic nerves of wild-type and Col6a1 ${ }^{-1-}$ mice under uninjured conditions and at 7 days post-crush. Right panel Densitometric quantification of CD68 vs. actin as determined by three independent Western blot experiments. Values for uninjured wild-type nerves were arbitrarily set to 1 $(n=4 ; * P<0.05 ; * * P<0.01)$. dpi days post-injury, $W T$ wild type
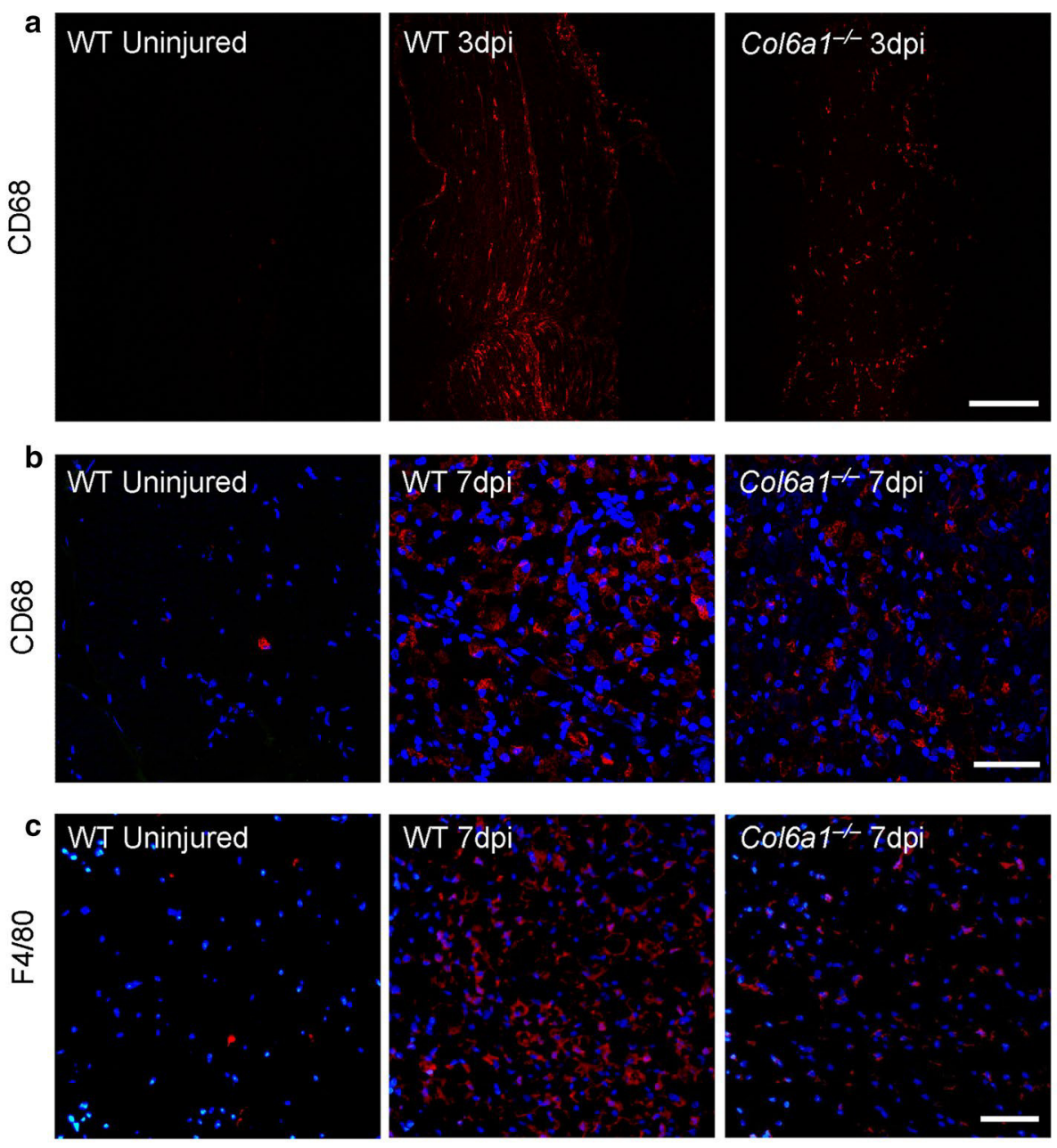

d

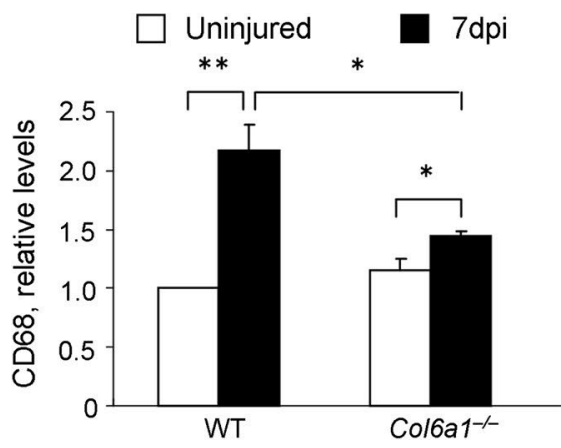

wild-type PMs in the absence or presence of purified collagen VI. Addition of collagen VI enhanced Arg-1 levels and reduced the levels of CD16 and iNOS in J774 macrophages (Supplementary Fig. S5a-c). Moreover, Western blot analysis for Arg-1 and PPAR $\gamma$ in PMs showed that both of these M2 markers were significantly increased upon treatment with purified collagen VI (Supplementary Fig. S5d, e). Taken together, these findings indicate that collagen VI promotes macrophage M2 polarization and exhibits an inhibitory effect on macrophage M1 polarization.
AKT and PKA are two key mediators of signaling pathways involved in macrophage polarization [3, 27, 38]. We thus evaluated whether collagen VI-induced macrophage polarization involves the activation of AKT and PKA signals. Western blot analysis showed that the phosphorylation of both AKT and PKA was increased upon stimulation with IL-4 in wild-type, but not in Col6al ${ }^{-/}$PMs (Supplementary Fig. S6a). Addition of collagen VI to BMDMs enhanced CD206 levels, which were inhibited by AKTi and H89 (Supplementary Fig. S6b). Moreover, immunofluorescence on J774 macrophages showed that collagen

\begin{tabular}{|l|lll|}
\hline Journal : Large 401 & Dispatch : 21-11-2014 & Pages : 17 \\
Article No : 1369 & $\square$ LE & $\square$ TYPESET \\
MS Code : ANEU-D-14-00635 & $\square \quad$ CP & $\square \quad$ DISK \\
\hline
\end{tabular}



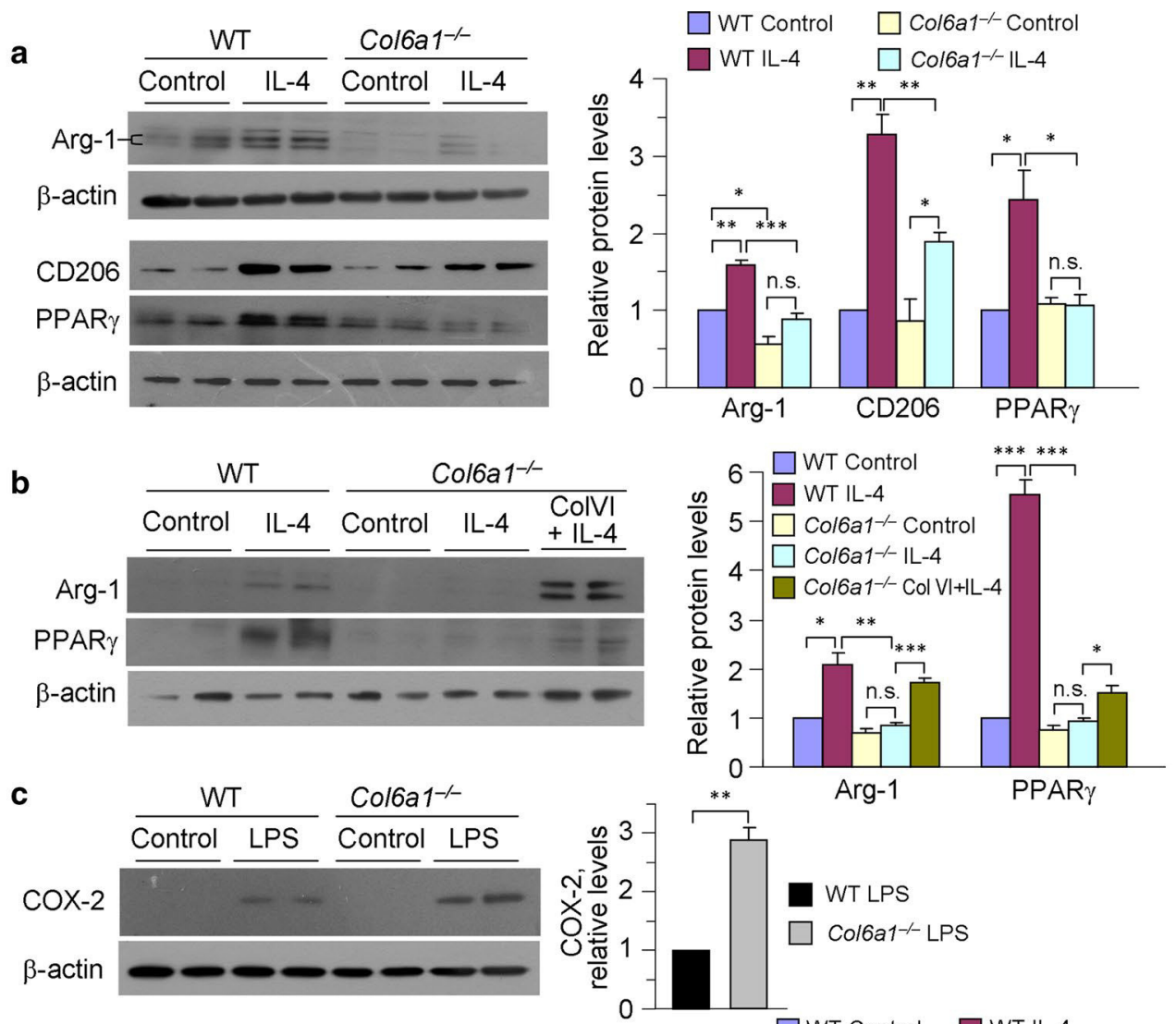

PPAR $\gamma$
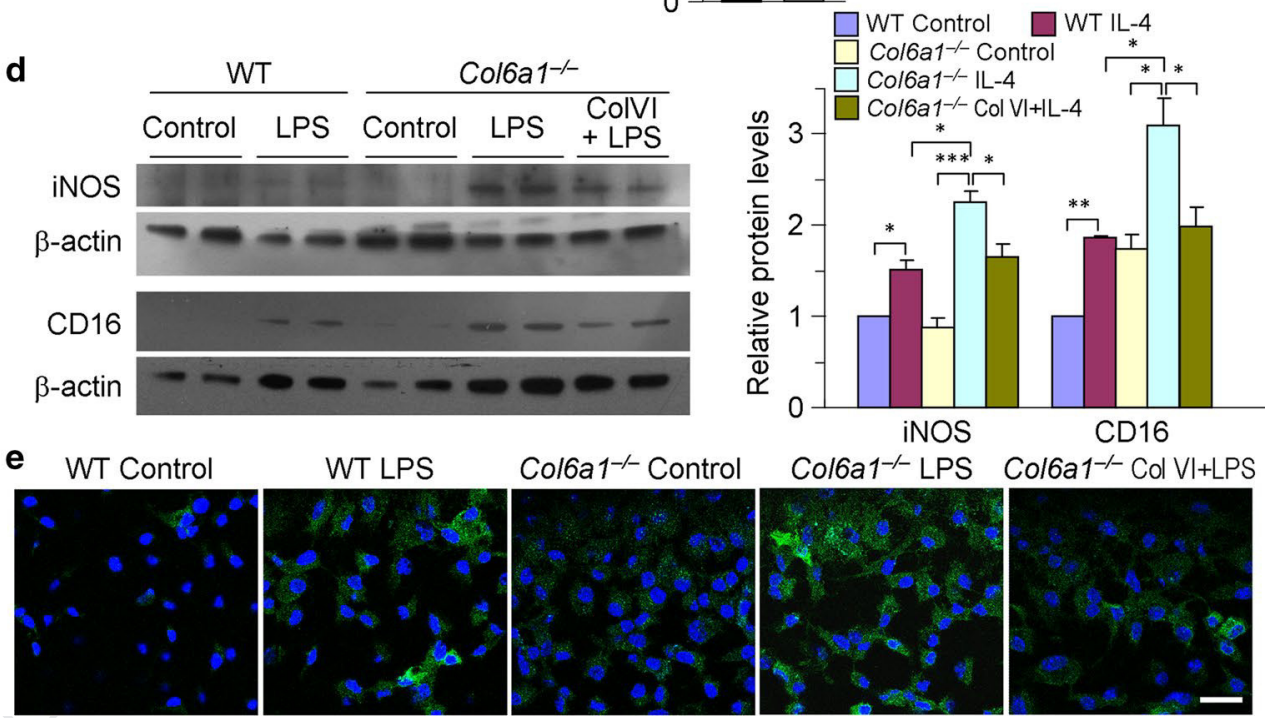

WT LPS

Col6a1 ${ }^{-1}$ Control
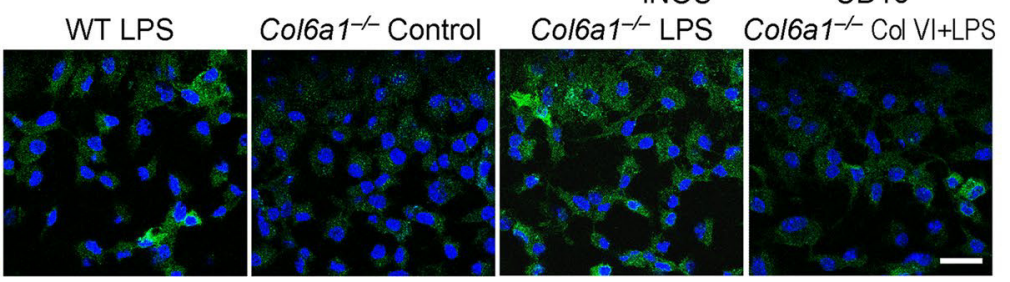

VI-promoted PPAR $\gamma$ expression was attenuated by AKTi and H89 (Supplementary Fig. S6c). These data support a role for the AKT and PKA pathways in modulating collagen VI-related macrophage M2 polarization.

Lack of collagen VI impairs macrophage M2 polarization in vivo after peripheral nerve injury

Next, we investigated the in vivo role of collagen VI in macrophage polarization in injured nerves. CD16 protein levels were higher in $\mathrm{Col}_{6 \mathrm{al}^{-/-}}$nerves than in wild-type nerves at 7 days post-crush (Fig. 6a). Furthermore, the expression of M2 marker genes Argl and Mrcl was upregulated in wildtype nerves, but not in $\mathrm{Col}_{6} \mathrm{al}^{-/-}$nerves at 7 days after crush (Fig. 6b, c). Immunofluorescence for CD206 showed that the amount of M2 macrophages in Col6al ${ }^{-/-}$nerves was dramatically lower than in wild-type nerves at 7 days post-injury (Fig. 6d). Western blot analysis confirmed that the levels of CD206 were significantly increased in wild-type nerves, but not in $\mathrm{Colbal}^{-/-}$nerves, at 7 days after crush when

\begin{tabular}{|l|lll|}
\hline & Journal : Large 401 & Dispatch : 21-11-2014 & Pages : 17 \\
Article No : 1369 & $\square$ LE & $\square$ TYPESET \\
MS Code : ANEU-D-14-00635 & $\square \quad$ CP & $\square \quad$ DISK \\
\hline
\end{tabular}


4 Fig. 5 Effect of collagen VI on macrophage polarization. a Left panel Western blot analysis for Arg-1, CD206 and PPAR $\gamma$ in wildtype and Col6a1 ${ }^{-1-}$ BMDMs under control conditions or following induction with IL-4. Right panel Densitometric quantification of Arg-1 vs. actin, CD206 vs. actin and PPAR $\gamma$ vs. actin, as determined by three independent Western blot experiments. Values for the wild-type control group were arbitrarily set to $1(n=4 ; * P<0.05$; $* * P<0.01 ; * * * P<0.001 ;$ n.s. not significant). b Left panel Western blot analysis for Arg-1 and PPAR $\gamma$ in wild-type, Col6a1 ${ }^{-1-}$ and collagen VI-coated $\left(5 \mu \mathrm{g} / \mathrm{cm}^{2}\right)$ Col6a1 ${ }^{-1-}$ PMs under control conditions or following induction with IL-4. Right panel Densitometric quantification of Arg-1 vs. actin and PPAR $\gamma$ vs. actin, as determined by three independent Western blot experiments. Values for the wildtype control group were arbitrarily set to 1 ( $n=3-4$; $* P<0.05$; $* * P<0.01 ; * * * P<0.001 ;$ n.s. not significant). c Left panel Western blot analysis for COX-2 in wild-type and Col6a1 ${ }^{-1-}$ PMs under control conditions or following induction with LPS. Right panel Densitometric quantification of COX-2 vs. actin, as determined by three independent Western blot experiments. Only LPS-induced COX-2 levels were calculated because of the extremely low COX-2 levels at baseline. Values for the wild-type LPS group were arbitrarily set to 1 $(n=3 ; * * P<0.01)$. d Left panel Western blot analysis for iNOS and CD16 in wild-type, Col6al ${ }^{-/-}$and collagen VI-coated $\left(5 \mu \mathrm{g} / \mathrm{cm}^{2}\right)$ Col6a1 $^{-1-}$ BMDMs under control conditions or following induction with LPS. Right panel Densitometric quantification of iNOS vs. actin and CD16 vs. actin, as determined by three independent Western blot experiments. Values for the wild-type control group were arbitrarily set to $1(n=3-4 ; * P<0.05 ; * * P<0.01 ; * * * P<0.001)$. e Immunofluorescence for COX-2 in wild-type, Col6a1 ${ }^{-1-}$ and collagen VIcoated $\left(5 \mu \mathrm{g} / \mathrm{cm}^{2}\right)$ Col6al ${ }^{-1-}$ BMDMs under control conditions or following induction with LPS. Scale bar $25 \mu \mathrm{m}$. Col VI collagen VI, $W T$ wild type compared to uninjured nerves (Fig. 6e). Finally, we normalized CD206 levels to CD68 levels and found that the relative amounts of CD206-positive M2 macrophages at 7 days postinjury were significantly lower in $\mathrm{Col} \mathrm{al} \mathrm{I}^{-/}$nerves than in wild-type nerves (Fig. 6f). Taken together, these data point to an impairment of macrophage M2 polarization in injured Col6al $^{-1-}$ nerves.

To assess the in vivo role of AKT and PKA pathways in modulating collagen VI-mediated macrophage M2 polarization, we analyzed the activation of these two signals in injured wild-type and Col6al ${ }^{-1-}$ nerves. At 7 days postcrush, phosphorylation of both AKT and PKA was markedly enhanced in wild-type nerves, but not in $\mathrm{Col}_{6} \mathrm{al}^{-/-}$nerves (Supplementary Fig. S7a, b). These findings support a role for the AKT and PKA pathways in the impaired macrophage M2 polarization and PNS regeneration of Col6al ${ }^{-1-}$ mice.

\section{Collagen VI-regulated macrophage function contributes} to PNS regeneration

To further confirm that the modulation of macrophage activities by collagen VI is crucial for PNS regeneration, we used macrophage-depleted in vivo models by injection of clodronate liposomes in wild-type and Col6a1 ${ }^{-/-}$ mice. Immunofluorescence for CD68 and F4/80 showed that injection of clodronate liposomes effectively depleted macrophages in sciatic nerves after crush (Supplementary Fig. S8). In the control group treated with PBS liposomes, the sciatic functional index was lower in $\mathrm{Col6a1}^{-/-}$mice than in wild-type mice, as expected (Fig. 7a). Following macrophage depletion by clodronate liposomes, the sciatic functional index was similarly reduced in wild-type and Col6al $^{-/-}$mice, thus indicating that the difference between the two genotypes was abolished after macrophage depletion (Fig. 7a). Moreover, the response to toe pinching was similar between wild-type and Col6al ${ }^{-/-}$mice after macrophage depletion with clodronate liposomes, but was delayed when compared to control treatment with PBS liposomes (Fig. 7b). Similarly, analysis of the toe spreading reflex showed that depletion of macrophages significantly delayed the toe extension in both wild-type and Col6a1 $1^{-/-}$ mice, and it abolished the difference between the two genotypes (Supplementary Table S2). Toluidine blue staining of sciatic nerve cross sections showed that in mice treated with PBS liposomes the number of myelinated axons was significant higher in wild-type nerves than in Col6al ${ }^{-/-}$ nerves at 21 days post-injury, as expected (Fig. 7c). However, when mice were treated with clodronate liposomes, the difference in myelinated axon number between the two genotypes was completely abolished (Fig. 7c). These results indicate that defective macrophage recruitment is the main cause for the delayed PNS regeneration of collagen VI-deficient mice.

To directly investigate whether the delayed PNS regeneration of $\mathrm{Colba1}^{-/-}$mice is due to the defects of collagen VI-regulated macrophage activities, we transplanted wild-type bone marrow cells into lethally irradiated wildtype mice (WT-WT) or collagen VI-deficient mice (WT$\left.\mathrm{Col}_{6} \mathrm{I}^{-/-}\right)$. Functional studies showed that there were no significant differences between WT-WT and WT-Col6a1 ${ }^{-/-}$ mice in the sciatic functional index score (Fig. 8a), time to initial response to toe pinch in digits 3,4 and 5 (Fig. 8b) and time to initial toe extension (Fig. 8c), indicating that the delayed PNS regeneration in Col6al ${ }^{-/-}$mice is rescued by transplantation of wild-type bone marrow cells. Next, we investigated whether the transplanted wild-type cells were able to rescue the decreased macrophage recruitment and polarization of $\mathrm{Col}_{6 \mathrm{al}} \mathrm{I}^{-/}$mice after nerve crush injury. Immunofluorescence for CD68 and F4/80 showed comparable CD68- and F4/80-positive macrophages in sciatic nerves of WT-WT and WT-Col6a1 ${ }^{-/-}$mice at 7 days postinjury (Fig. 8d). Western blot analysis showed that CD206 levels were similar between WT-WT and WT-Col6a1 ${ }^{-/-}$ mice at 7 days post-injury (Fig. 8e). Taken together, these findings provide evidence demonstrating that the delayed PNS regeneration in Col6al ${ }^{-/-}$mice is induced by the deficits in macrophage migration and polarization. 
a

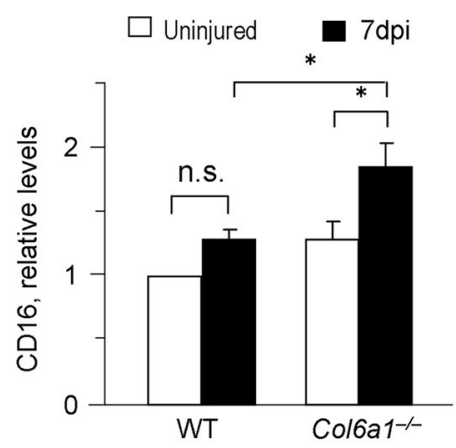

b

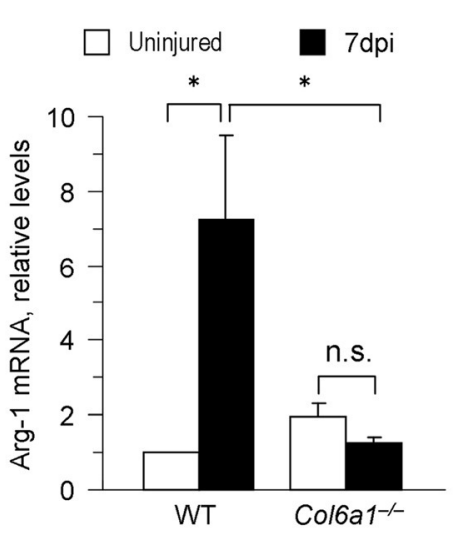

C

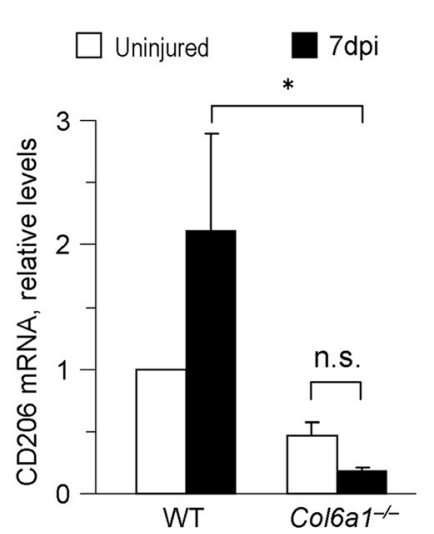

d

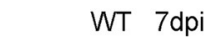

e

$$
\frac{\text { WT }}{\text { Uninjured } 7 \mathrm{dpi}} \frac{\text { Col6a1 }}{\text { Uninjured }} 7 \mathrm{dpi}
$$
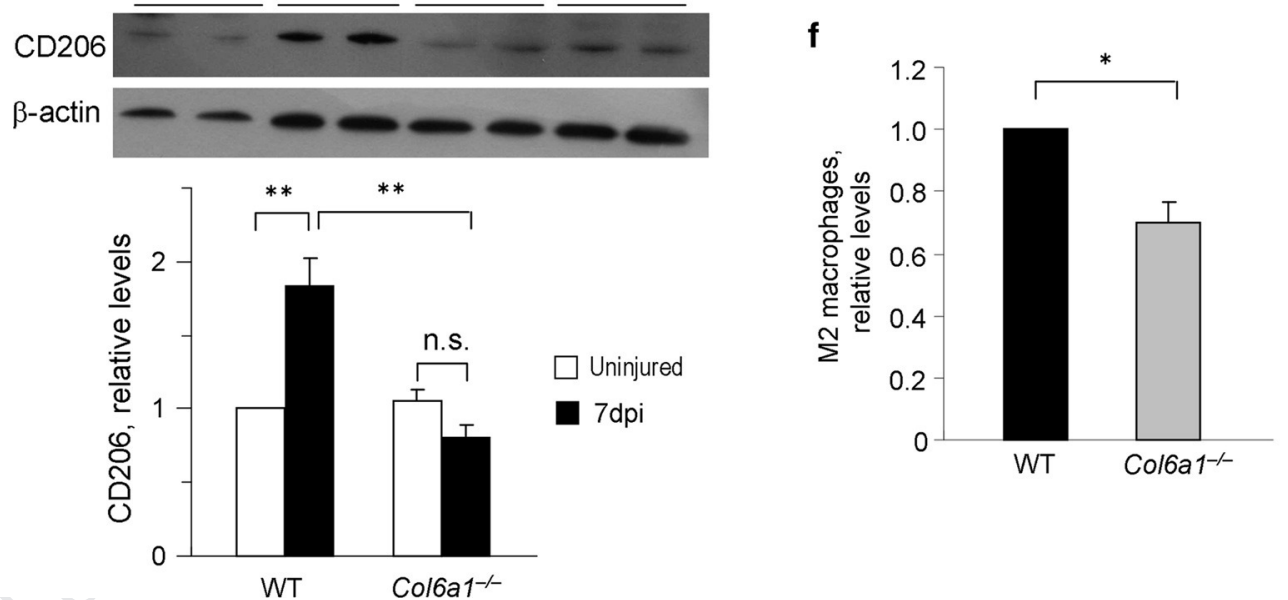

Fig. 6 Ablation of collagen VI decreases macrophage M2 polarization after nerve injury. a Left panel Western blot analysis for CD16 in sciatic nerves from wild-type and Col6a1 ${ }^{-1-}$ mice under uninjured conditions and at 7 days post-crush. Right panel Densitometric quantification of CD16 vs. actin, as determined by three independent Western blot experiments. Values for uninjured wild-type nerves were arbitrarily set to 1 ( $n=4 ; * P<0.05 ; n . s$. not significant). Real-time RT-PCR analysis for Arg-1 (b) and CD206 (c) mRNA in sciatic nerves from wild-type and Col6a1 ${ }^{-1-}$ mice under uninjured conditions and at 7 days post-crush. Values for uninjured wild-type nerves were arbitrarily set to 1 . GAPDH was used as a reference gene $(n=3-5 ; * P<0.05 ;$ n.s. not significant). d Immunofluores- cence for CD206 in cross sections of sciatic nerves from wild-type and Col6a1 ${ }^{-1-}$ mice at 7 days post-crush. Scale bar $25 \mu \mathrm{m}$. e Top panel Western blot analysis for CD206 in sciatic nerves from wildtype and Col6a1 $1^{-1-}$ mice under uninjured conditions and at 7 days post-crush. Bottom panel Densitometric quantification of CD206 vs. actin as determined by three independent Western blot experiments. Values for uninjured wild-type nerves were arbitrarily set to $1(n=4$; $* * P<0.01 ; n . s$. not significant). f Quantification of CD206 vs. CD68 in sciatic nerves from wild-type and Col6al ${ }^{-1-}$ mice at 7 days postcrush as determined by three independent Western blot experiments. Values for uninjured wild-type nerves were arbitrarily set to $1(n=4$; $* * P<0.05)$. dpi days post-injury, $W T$ wild type

\begin{tabular}{|llll|} 
Journal : Large 401 & Dispatch : 21-11-2014 & Pages : 17 \\
Article No : $\mathbf{1 3 6 9}$ & $\square$ LE & $\square$ TYPESET \\
MS Code : ANEU-D-14-00635 & $\square \quad C P$ & $\square$ DISK \\
\hline
\end{tabular}


Fig. 7 Macrophage depletion leads to similar regenerative responses in wild-type and collagen VI-deficient peripheral nerves. a Quantification of the sensory-motor function of wild-type and Col6a1 ${ }^{-1-}$ mice under control conditions (PBS liposomes) and after macrophage depletion (clodronate liposomes) by analyzing the sciatic functional index from footprint tracks before crush and at 7, 11, 14 and 17 days post-crush $(n=5-7 ; * P<0.05$ and $* * P<0.01$, Col6a1 ${ }^{-1-}$ PBS vs. wild-type PBS; ${ }^{\wedge} P<0.05$, ${ }^{\wedge \wedge} P<0.01$ and ${ }^{\wedge \wedge} P<0.001$, wild-type clodronate vs. wild-type PBS; ${ }^{\#} P<0.05$ and ${ }^{\# \#} P<0.01$, Col6a1 ${ }^{-1-}$ clodronate vs. Col6a1 ${ }^{-1-}$ PBS). b Quantification of the sensory function of wild-type and Col6a1 ${ }^{-1-}$ mice under control conditions (PBS liposomes) and after macrophage depletion (clodronate liposomes) by recording the initial response time to the pinch using forceps in the digits 3,4 and 5 after sciatic nerve crush $(n=5-7$; $* P<0.05 ;$ n.s. not significant) c Representative images of toluidine blue staining and morphometric analysis of the myelinated axon number in cross sections of injured sciatic nerves at 21 days post-crush from wild-type and $\mathrm{Col}_{6 a 1^{-1-}}$ mice that received PBS liposomes or clodronate liposomes. Scale bar $40 \mu \mathrm{m}(n=3 ; * * * P<0.001$; n.s. not significant). dpi days post-injury, $W T$ wild type
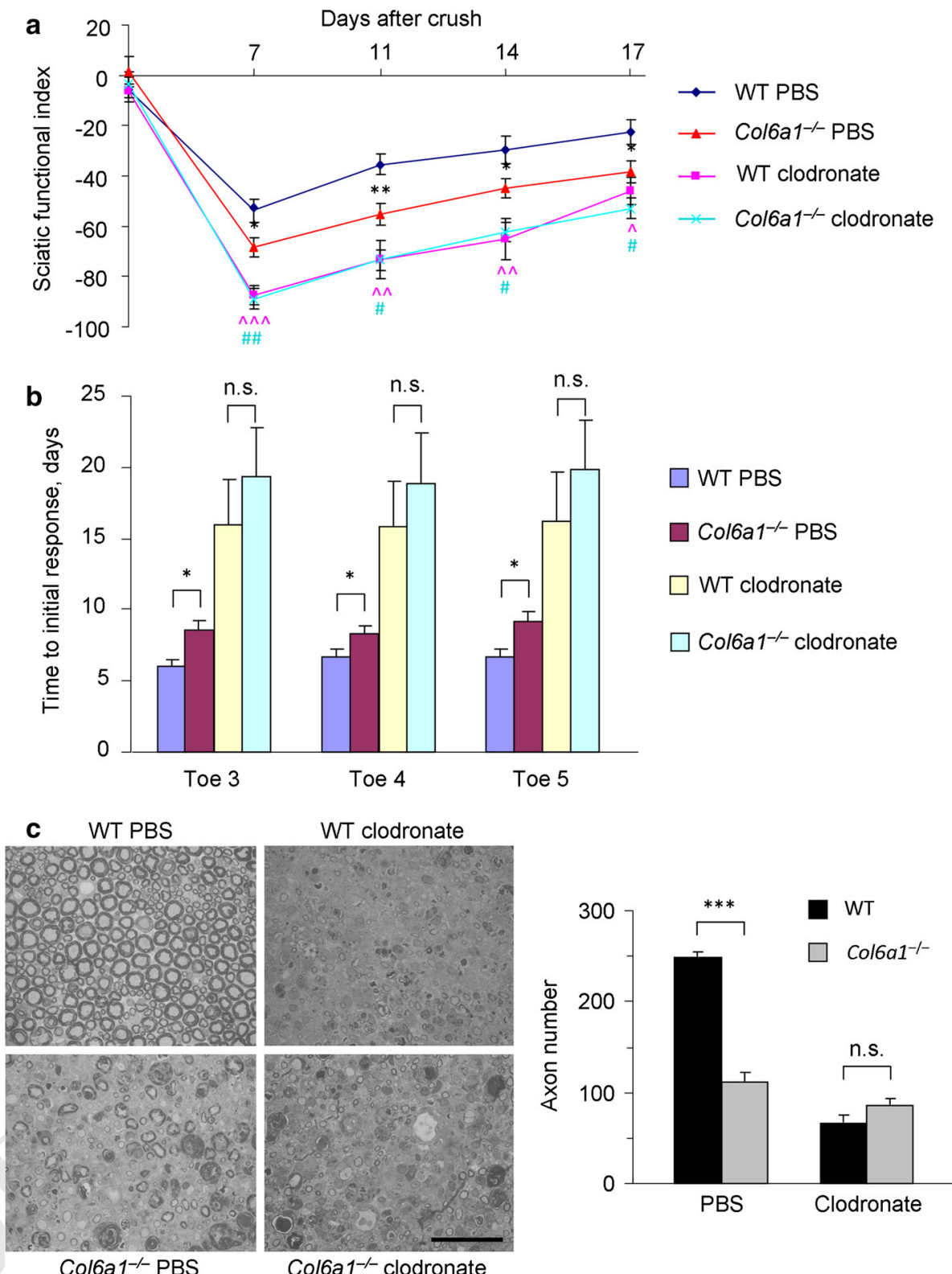

Col6a1 ${ }^{-1}$ PBS

\section{Discussion}

Our previous work demonstrated that collagen VI is required for muscle regeneration [46], suggesting a role in tissue repair. The results presented in this study show that collagen VI promotes PNS regeneration by regulating macrophage recruitment and polarization. Lack of collagen VI in Col6al ${ }^{-/-}$mice prevents macrophage recruitment and phenotypic transition after sciatic nerve crush, which in turn inhibits PNS regeneration. Previous studies showed that macrophages play a pivotal function in Wallerian degeneration by clearing myelin debris and in axonal regeneration by secreting a variety of soluble factors [33]. However, the molecular mechanisms underlying macrophage recruitment into injured nerves are not well understood. It is well accepted that soluble factors secreted by the disrupted axon/Schwann cell nerve unit are responsible for macrophage recruitment following nerve injury [33, 45]. Among these factors, IL- $1 \beta$ and MCP-1 are two major macrophage chemoattractants in injured peripheral nerves [36, 41, 45]. However, blockade of IL-1 $\beta$ and MCP-1 with function-blocking antibodies does not completely inhibit macrophage recruitment into injured peripheral nerves in vivo [36]. Similar effects were also displayed by in vitro experiments, where addition of MCP-1 neutralizing antibodies to conditioned media from Schwann cell cultures and nerve segments does not completely block macrophage migration [45]. These findings indicate that other chemoattractants are also secreted by the injured peripheral nerves. In the current study, we found that collagen VI promotes

\begin{tabular}{|l|lll|}
\hline Journal : Large 401 & Dispatch : 21-11-2014 & Pages : 17 \\
Article No : 1369 & $\square$ LE & $\square$ TYPESET \\
MS Code : ANEU-D-14-00635 & $\square \quad C P$ & $\square$ DISK \\
\hline
\end{tabular}


a

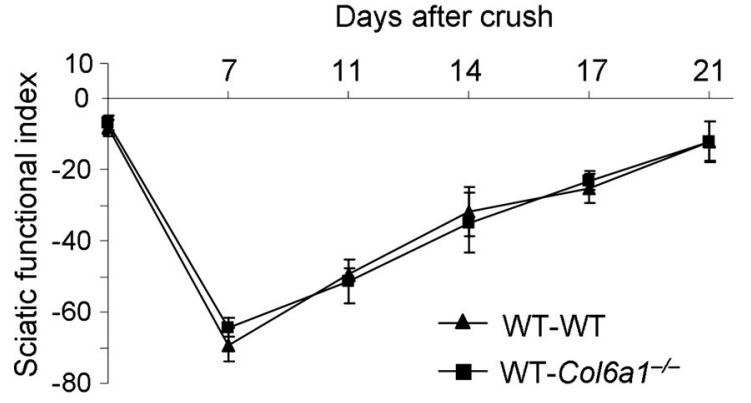

b

Toe pinch

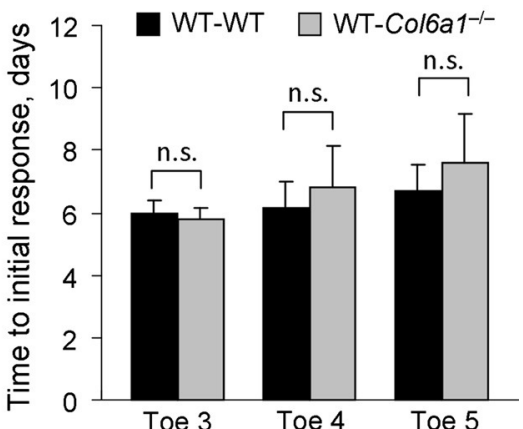

Toe spread reflex

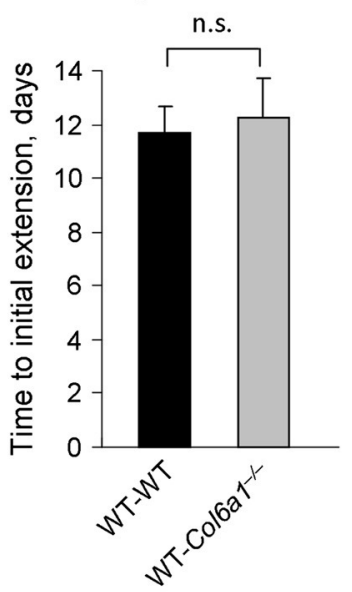

e

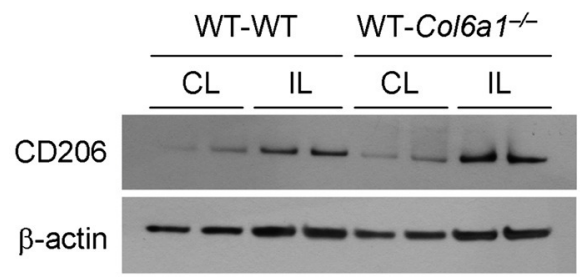

d
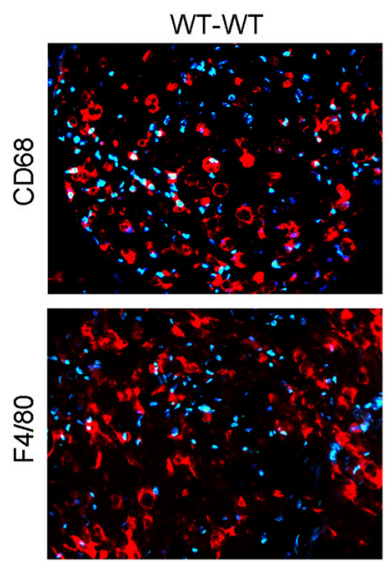

WT-Col6a1 ${ }^{-1-}$
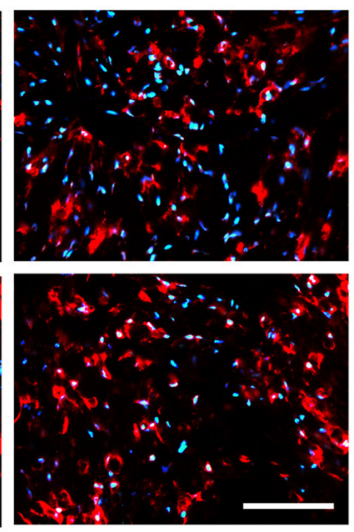

Fig. 8 Transplantation of wild-type bone marrow cells into Col6 $\mathrm{II}^{-1-}$ host mice rejuvenates regeneration and macrophage activities after nerve injury. a Quantification of sensory-motor function of wild-type bone marrow cells transplanted into wild-type mice (WTWT) and of wild-type bone marrow cells transplanted into Col6a1 ${ }^{-1-}$ mice (WT-Col6a1 ${ }^{-l-}$ ) by analyzing the sciatic functional index from footprint tracks before crush and at 7, 11, 14, 17 and 21 days postcrush $(n=5-7)$. b Quantification of sensory function of WT-WT and WT-Col6a1 ${ }^{-1-}$ mice after sciatic nerve crush by recording the initial response time (day post-injury) to the pinch using forceps in the digits 3, 4 and 5 ( $n=5-7$; n.s. not significant). c Quantification of motor function of WT-WT and WT-Col6a1 ${ }^{-1-}$ mice after sciatic nerve crush by recording the initial extension time (day post-injury)

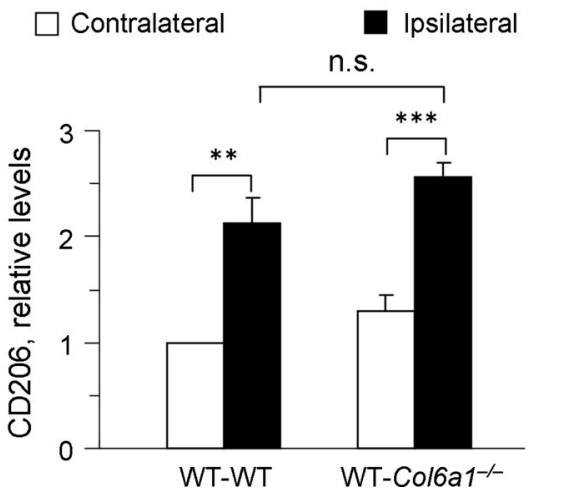

to the toe spreading reflex ( $n=4-6$; n.s. not significant). d Immunofluorescence for CD68 and F4/80 in cross sections of injured sciatic nerves from WT-WT and WT-Col6a1 ${ }^{-1-}$ mice at 7 days post-crush. Scale bar $50 \mu \mathrm{m}$. e Left panel Western blot analysis for CD206 in sciatic nerves from WT-WT and WT-Col6a1 ${ }^{-1-}$ mice at 7 days postcrush. Right panel Densitometric quantification of CD206 vs. actin as determined by three independent Western blot experiments. Values for uninjured WT-WT contralateral nerves were arbitrarily set to 1 ( $n=4 ; * * P<0.01 ; * * * P<0.001 ; n . s$. not significant). $C L$ contralateral, IL ipsilateral, WT-Col6a1 ${ }^{-/}$wild-type bone marrow cells transplanted into Col6al ${ }^{-1-}$ mice, WT-WT wild-type bone marrow cells transplanted into wild-type mice

\begin{tabular}{llll|}
\hline Journal : Large 401 & Dispatch : 21-11-2014 & Pages : 17 \\
Article No : $\mathbf{1 3 6 9}$ & $\square$ LE & $\square$ TYPESET \\
MS Code : ANEU-D-14-00635 & $\square \quad C P$ & $\square$ DISK \\
\hline
\end{tabular}


macrophage migration both in vitro and in vivo and that Col6a1 ${ }^{-/-}$macrophages exhibit a reduced migration capability in the Matrigel plug assay. In the sciatic nerve crush model, macrophage recruitment was markedly impaired in Col6a1 $1^{-/}$nerves. Thus, we identified collagen VI as a novel chemoattractant that triggers macrophage recruitment into injured nerves.

Our recent work demonstrated that in peripheral nerves collagen VI is abundantly deposited by Schwann cells and macrophages, but not by axons [7]. Upon injury in the PNS, Schwann cells dedifferentiate to a progenitor/ stem cell-like state [35], expressing high levels of collagen VI [48]. In this study, we found that the expression of collagen VI is significantly upregulated upon sciatic nerve injury. On the one hand, this enhancement is likely contributed by the dedifferentiated Schwann cells; on the other hand, it is related to the increased number of macrophages after injury. In this regard, it is plausible that at the initial stage the dedifferentiated Schwann cells are responsible for increasing collagen VI deposition in injured nerves, which in turn promotes macrophage recruitment in a paracrine manner. Thereafter, both paracrine and autocrine effects may exist for the collagen VI contribution to macrophage recruitment. Our findings demonstrate that the impaired macrophage recruitment in injured $\mathrm{Col}_{6 \mathrm{al}}{ }^{-/-}$ nerves is rescued by transplantation of wild-type bone marrow cells, highlighting the autocrine effect of collagen VI in macrophage recruitment after peripheral nerve injury. Our data indicate that collagen VI acts as a chemoattractant for macrophages, a finding that is fully consistent with previous studies showing that certain ECM proteins, such as fibronectin, laminin and collagen IV, exhibit specific chemoattractant activities for different cells $[1,24,49]$. In addition to directly exhibiting chemotactic activity, collagen VI also influence the expression of other chemoattractants. For example, we found in this study that the upregulation of IL- $1 \beta$ and MCP-1 induced by sciatic nerve crush injury is significantly impaired in $\mathrm{Col}_{6 \mathrm{al}^{-/}}$mice, suggesting that collagen VI is able to promote the recruitment of macrophages into the injured nerves through a variety of molecular mechanisms.

The function of macrophages in PNS regeneration is also related to their phenotype, where M2 macrophages stimulate regeneration [30]. Therefore, macrophage polarization from the M1 to M2 phenotype is crucial for successful PNS regeneration. It has been shown that acute peripheral nerve injury elicits an M2 macrophage response [50]. However, the mechanisms that trigger and modulate macrophage polarization are not well understood. To date, it is known that macrophage polarization is largely controlled by a small group of signals and factors, such as nuclear factor $\kappa \mathrm{B}(\mathrm{NF}-\kappa \mathrm{B})$, mammalian target of rapamycin (mTOR), signal transducer and activator of transcription 6 (STAT6),
PPAR $\gamma$, Kruppel-like factor 4 (KLF4), AKT and PKA [4, $25,27,38]$. In this study, we identified collagen VI as a novel factor regulating macrophage polarization. We found that addition of collagen VI promotes the J774 macrophage polarized toward the M2 phenotype. In the light of these findings, we isolated primary BMDMs and PMs from wildtype and Col6al ${ }^{-/}$mice and stimulated their polarization into M1 and M2 phenotypes with LPS and IL-4, respectively. Consistent with our hypothesis, deficiency of collagen VI impairs macrophage M2 polarization and promotes macrophage M1 polarization, which can be reversed by addition of purified native collagen VI.

Our findings indicate that collagen VI plays a pivotal role in macrophage polarization. Although one recent in vitro work showed that some specific ECM components, namely collagen I and fibronectin, are not needed for macrophage polarization [37], previous evidence suggests that ECM plays a key role in this process. For example, ECM-derived biologic scaffolds induce an in vivo constructive tissue remodeling by promoting an M2 macrophage response [42]. In a myocardial infarction model, deficiency of matrix metalloproteinase 28 (MMP-28) was found to attenuate macrophage M2 polarization and reduce the expression of several ECM genes [28]. Among the different ECM molecules, collagen VI seemed to be the best candidate for regulating macrophage polarization. M2 macrophages produce higher levels of collagen VI than M1 macrophages [40]. Moreover, macrophages in the adipose tissue of insulin-resistant subjects are associated with collagen VI deposition and exhibit M2 phenotype [44]. These indirect observations prompted us to investigate the role of this ECM molecule for macrophage polarization. In addition to in vitro data, we obtained direct in vivo evidence showing that collagen VI regulates macrophage polarization during PNS regeneration. We found that at 7 days postinjury, the levels of M2 macrophages were decreased in Colbal $^{-/-}$nerves, indicating an impairment of macrophage skewing, which in turn inhibits PNS regeneration.

Notably, the different nerve regeneration response of wildtype and $\mathrm{Col}_{\mathrm{Ca}} \mathrm{I}^{-/-}$mice is abolished by in vivo macrophage depletion. Moreover, our data demonstrate that the defective PNS regeneration of $\mathrm{Colbal}^{-/-}$mice, and the defective injury-induced macrophage migration and polarization, are rescued by transplantation of wild-type bone marrow cells. Together, these findings highlight the mechanistic insight of collagen VI regulation of macrophage activities as a critical player for PNS regeneration. In addition, we provide evidence showing that the AKT and PKA pathways contribute to collagen VI-regulated macrophage function. Addition of collagen VI to cultured macrophages promotes the activation of AKT and PKA, whereas collagen VI ablation abolishes IL-4-induced activation of both signals. Blockade of AKT and PKA by their inhibitors abrogates collagen VI-induced 
macrophage migration and polarization. Furthermore, our in vivo data indicate that the increased activation of AKT and PKA in injured nerves is completely inhibited by collagen VI ablation. Our results on the one hand support the concept that AKT and PKA pathways are necessary for macrophage migration and polarization $[3,10,11,27,38]$ and axonal regeneration $[9,17]$; on the other hand, they provide insights into the downstream targets of collagen VI-regulated macrophage function in PNS regeneration.

In summary, we demonstrate in this study that collagen VI is a pivotal factor for macrophage function. In this context, besides providing novel molecular understanding for macrophage migration and polarization, our study points out potentially broad implications for collagen VI in inflammatory diseases. These data also provide evidence for a beneficial impact of collagen VI on peripheral nerve regeneration via modulation of macrophage activities. In addition to contributing to the understanding of the roles of collagen VI in the experimental setting of PNS regeneration, our findings might have useful implications for clinical study. For example, it is reasonable that application of collagen VI as a coating substrate for the artificial nerve guide conduits may be beneficial for improving the peripheral nerve functional recovery in patients. Future studies will allow testing this possibility and evaluating the effectiveness of such regenerative approaches.

Acknowledgments We are grateful to W. Giuriati for technical assistance, P. Braghetta for helping with mice and R. Wagener for providing $\alpha 3(\mathrm{VI})$ collagen antibodies. This work was supported by grants from the Telethon Foundation (GGP10225 and GGP11082), the Italian Ministry of Education, University and Research (RBAP11Z3YA_003), and the University of Padua Strategic Projects. P. Chen is supported by a fellowship from the Cariparo Foundation and an ImmunoTools award providing the cytokines used in this study.

Conflict of interest The authors declare no potential conflicts of interest.

\section{References}

1. Aznavoorian S, Stracke ML, Krutzsch H et al (1990) Signal transduction for chemotaxis and haptotaxis by matrix molecules in tumor cells. J Cell Biol 10:1427-1438

2. Bonaldo P, Braghetta P, Zanetti M et al (1998) Collagen VI deficiency induces early onset myopathy in the mouse: an animal model for Bethlem myopathy. Hum Mol Genet 7:2135-2140

3. Byles V, Covarrubias AJ, Ben-Sahra I et al (2013) The TSC-mTOR pathway regulates macrophage polarization. Nat Commun 4:2834

4. Chen P, Bonaldo P (2013) Role of macrophage polarization in tumor angiogenesis and vessel normalization: implications for new anticancer therapies. Int Rev Cell Mol Biol 301:1-35

5. Chen P, Cescon M, Bonaldo P (2013) Collagen VI in cancer and its biological mechanisms. Trends Mol Med 19:410-417

6. Chen P, Cescon M, Bonaldo P (2014) Autophagy-mediated regulation of macrophages and its applications for cancer. Autophagy 10:192-200
7. Chen P, Cescon M, Megighian A, Bonaldo P (2014) Collagen VI regulates peripheral nerve myelination and function. FASEB J 28:1145-1156

8. Chen P, Huang Y, Bong R et al (2011) Tumor-associated macrophages promote angiogenesis and melanoma growth via adrenomedullin in a paracrine and autocrine manner. Clin Cancer Res 17:7230-7239

9. Christie KJ, Webber CA, Martinez JA et al (2010) PTEN inhibition to facilitate intrinsic regenerative outgrowth of adult peripheral axons. J Neurosci 30:9306-9315

10. Cote SC, Pasvanis S, Bounou S, Dumais N (2009) CCR7-specific migration to CCL19 and CCL21 is induced by PGE(2) stimulation in human monocytes: involvement of $\mathrm{EP}(2) / \mathrm{EP}(4)$ receptors activation. Mol Immunol 46:2682-2693

11. Diaz-Munoz MD, Osma-Garcia IC, Iniguez MA, Fresno M (2013) Cyclooxygenase-2 deficiency in macrophages leads to defective p110gamma PI3K signaling and impairs cell adhesion and migration. J Immunol 191:395-406

12. Du R, Lu KV, Petritsch $\mathrm{C}$ et al (2008) HIF1alpha induces the recruitment of bone marrow-derived vascular modulatory cells to regulate tumor angiogenesis and invasion. Cancer Cell 13:206-220

13. Dubovy P, Jancalek R, Kubek T (2013) Role of inflammation and cytokines in peripheral nerve regeneration. Int Rev Neurobiol 108:173-206

14. Farah MH, Pan BH, Hoffman PN et al (2011) Reduced BACE1 activity enhances clearance of myelin debris and regeneration of axons in the injured peripheral nervous system. J Neurosci 31:5744-5754

15. Gara SK, Grumati P, Squarzoni S et al (2011) Differential and restricted expression of novel collagen VI chains in mouse. Matrix Biol 30:248-257

16. Germano G, Frapolli R, Belgiovine C et al (2013) Role of macrophage targeting in the antitumor activity of trabectedin. Cancer Cell 23:249-262

17. Ghosh-Roy A, Wu Z, Goncharov A et al (2010) Calcium and cyclic AMP promote axonal regeneration in Caenorhabditis elegans and require DLK-1 kinase. J Neurosci 30:3175-3183

18. Gordon S, Martinez FO (2010) Alternative activation of macrophages: mechanism and functions. Immunity 32:593-604

19. Gordon S, Taylor PR (2005) Monocyte and macrophage heterogeneity. Nat Rev Immunol 5:953-964

20. Griffin JW, George R, Ho T (1993) Macrophage systems in peripheral nerves. A review. J Neuropathol Exp Neurol 52:553-560

21. Horie H, Kadoya T, Hikawa N et al (2004) Oxidized galectin-1 stimulates macrophages to promote axonal regeneration in peripheral nerves after axotomy. J Neurosci 24:1873-1880

22. Inserra MM, Bloch DA, Terris DJ (1998) Functional indices for sciatic, peroneal, and posterior tibial nerve lesions in the mouse. Microsurgery 18:119-124

23. Irwin WA, Bergamin N, Sabatelli P et al (2003) Mitochondrial dysfunction and apoptosis in myopathic mice with collagen VI deficiency. Nat Genet 35:367-371

24. Klominek J, Robért KH, Sundqvist KG (1993) Chemotaxis and haptotaxis of human malignant mesothelioma cells: effects of fibronectin, laminin, type IV collagen, and an autocrine motility factor-like substance. Cancer Res 53:4376-4382

25. Liao X, Sharma N, Kapadia F et al (2011) Kruppel-like factor 4 regulates macrophage polarization. J Clin Invest 121:2736-2749

26. López-Vales R, Navarro X, Shimizu T et al (2008) Intracellular phospholipase $\mathrm{A}(2)$ group IVA and group VIA play important roles in Wallerian degeneration and axon regeneration after peripheral nerve injury. Brain 131:2620-2631

27. Ma L, Dong F, Zaid M et al (2012) ABCA1 protein enhances toll-like receptor 4 (TLR4)-stimulated interleukin-10 (IL-10)

\begin{tabular}{|llll|} 
Journal : Large 401 & Dispatch : 21-11-2014 & Pages : 17 \\
Article No : $\mathbf{1 3 6 9}$ & $\square$ LE & $\square$ TYPESET \\
MS Code : ANEU-D-14-00635 & $\square \quad$ CP & $\square$ DISK \\
\hline
\end{tabular}


secretion through protein kinase A (PKA) activation. J Biol Chem 287:40502-40512

28. Ma Y, Halade GV, Zhang J et al (2013) Matrix metalloproteinase-28 deletion exacerbates cardiac dysfunction and rupture after myocardial infarction in mice by inhibiting M2 macrophage activation. Circ Res 112:675-688

29. Mantovani A, Biswas SK, Galdiero MR et al (2013) Macrophage plasticity and polarization in tissue repair and remodeling. J Pathol 229:176-185

30. Mokarram N, Merchant A, Mukhatyar V et al (2012) Effect of modulating macrophage phenotype on peripheral nerve repair. Biomaterials 33:8793-8801

31. Mosser DM, Edwards JP (2008) Exploring the full spectrum of macrophage activation. Nat Rev Immunol 8:958-969

32. Mueller M, Leonhard C, Wacker K et al (2003) Macrophage response to peripheral nerve injury: the quantitative contribution of resident and hematogenous macrophages. Lab Invest 83:175-185

33. Namikawa K, Okamoto T, Suzuki A et al (2006) Pancreatitisassociated protein-III is a novel macrophage chemoattractant implicated in nerve regeneration. J Neurosci 26:7460-7467

34. Niemi JP, DeFrancesco-Lisowitz A, Roldán-Hernández L et al (2013) A critical role for macrophages near axotomized neuronal cell bodies in stimulating nerve regeneration. J Neurosci 33:16236-16248

35. Parrinello S, Napoli I, Ribeiro S et al (2010) EphB signaling directs peripheral nerve regeneration through Sox2-dependent Schwann cell sorting. Cell 143:145-155

36. Perrin FE, Lacroix S, viles-Trigueros M, David S (2005) Involvement of monocyte chemoattractant protein-1, macrophage inflammatory protein-1alpha and interleukin-1beta in Wallerian degeneration. Brain 128:854-866

37. Ploeger DT, van Putten SM, Koerts JA et al (2012) Human macrophages primed with angiogenic factors show dynamic plasticity, irrespective of extracellular matrix components. Immunobiology 217:299-306

38. Santos-Sierra S, Deshmukh SD, Kalnitski J et al (2009) Mal connects TLR2 to PI3Kinase activation and phagocyte polarization. EMBO J 28:2018-2027

39. Schafer M, Fruttiger M, Montag D et al (1996) Disruption of the gene for the myelin-associated glycoprotein improves axonal regrowth along myelin in C57BL/Wlds mice. Neuron 16:1107-1113

40. Schnoor M, Cullen P, Lorkowski J et al (2008) Production of type VI collagen by human macrophages: a new dimension in macrophage functional heterogeneity. J Immunol 180:5707-5719
41. Shamash S, Reichert F, Rotshenker S (2002) The cytokine network of Wallerian degeneration: tumor necrosis factoralpha, interleukin-1alpha, and interleukin-1beta. J Neurosci 22:3052-3060

42. Sicari BM, Johnson SA, Siu BF et al (2012) The effect of source animal age upon the in vivo remodeling characteristics of an extracellular matrix scaffold. Biomaterials 33:5524-5533

43. Siconolfi LB, Seeds NW (2001) Mice lacking tPA, uPA, or plasminogen genes showed delayed functional recovery after sciatic nerve crush. J Neurosci 21:4348-4355

44. Spencer M, Yao-Borengasser A, Unal R et al (2010) Adipose tissue macrophages in insulin-resistant subjects are associated with collagen VI and fibrosis and demonstrate alternative activation. Am J Physiol Endocrinol Metab 299:E1016-E1027

45. Tofaris GK, Patterson PH, Jessen KR, Mirsky R (2002) Denervated Schwann cells attract macrophages by secretion of leukemia inhibitory factor (LIF) and monocyte chemoattractant protein- 1 in a process regulated by interleukin-6 and LIF. J Neurosci 22:6696-6703

46. Urciuolo A, Quarta M, Morbidoni V et al (2013) Collagen VI regulates satellite cell self-renewal and muscle regeneration. Nat Commun 4:1964

47. Vargas ME, Watanabe J, Singh SJ et al (2010) Endogenous antibodies promote rapid myelin clearance and effective axon regeneration after nerve injury. Proc Natl Acad Sci 107:11993-11998

48. Vitale P, Braghetta P, Volpin D et al (2001) Mechanisms of transcriptional activation of the col6a1 gene during Schwann cell differentiation. Mech Dev 102:145-156

49. Wang AZ, Chen JM, Fisher GW et al (1994) Improved in vitro models for assay of rheumatoid synoviocyte chemotaxis. Clin Exp Rheumatol 12:293-299

50. Ydens E, Cauwels A, Asselbergh B et al (2012) Acute injury in the peripheral nervous system triggers an alternative macrophage response. J Neuroinflammation 9:176

51. Zhang L, Johnson D, Johnson JA (2013) Deletion of Nrf2 impairs functional recovery, reduces clearance of myelin debris and decreases axonal remyelination after peripheral nerve injury. Neurobiol Dis 54:329-338

52. Zhu X, Lee JY, Timmins JM et al (2008) Increased cellular free cholesterol in macrophage-specific Abcal knock-out mice enhances pro-inflammatory response of macrophages. J Biol Chem 283:22930-22941

\begin{tabular}{|llll|} 
Journal : Large 401 & Dispatch : 21-11-2014 & Pages : 17 \\
Article No : $\mathbf{1 3 6 9}$ & $\square$ LE & $\square$ TYPESET \\
MS Code : ANEU-D-14-00635 & $\square \quad C P$ & $\square$ DISK \\
\hline
\end{tabular}


Journal: $\quad \mathbf{4 0 1}$

Article: $\quad 1369$

\section{包 Springer}

the language of science

\section{Author Query Form}

Please ensure you fill out your response to the queries raised below and return this form along with your corrections

Dear Author

During the process of typesetting your article, the following queries have arisen. Please check your typeset proof carefully against the queries listed below and mark the necessary changes either directly on the proof/online grid or in the 'Author's response' area provided below

\begin{tabular}{|l|l|l|}
\hline Query & Details Required & Author's Response \\
\hline AQ1 & $\begin{array}{l}\text { Kindly check and confirm "the recovery of sensory motor coordination [24]" 24 } \\
\text { mentioned here is cited as reference is correct and amend if necessary. }\end{array}$ & \\
\hline
\end{tabular}

